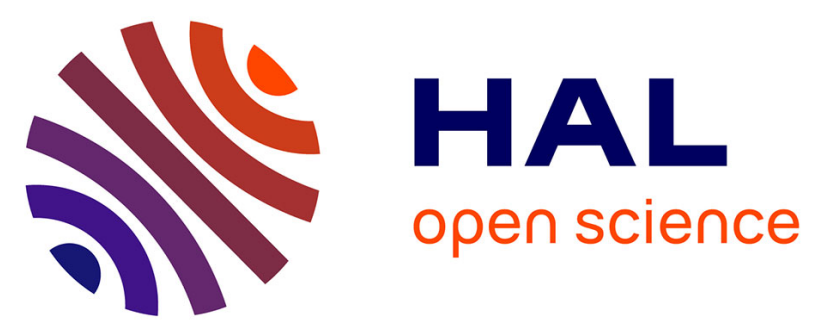

\title{
Domain decomposition methods and high-order absorbing boundary conditions for the numerical simulation of the time dependent Schrödinger equation with ionization and recombination by intense electric field
}

\author{
Xavier Antoine, Emmanuel Lorin, André D. Bandrauk
}

\section{To cite this version:}

Xavier Antoine, Emmanuel Lorin, André D. Bandrauk. Domain decomposition methods and highorder absorbing boundary conditions for the numerical simulation of the time dependent Schrödinger equation with ionization and recombination by intense electric field. Journal of Scientific Computing, 2015, 64 (3), pp.620-646. 10.1007/s10915-014-9902-5 . hal-01094831

\section{HAL Id: hal-01094831 \\ https://hal.science/hal-01094831}

Submitted on 9 Feb 2016

HAL is a multi-disciplinary open access archive for the deposit and dissemination of scientific research documents, whether they are published or not. The documents may come from teaching and research institutions in France or abroad, or from public or private research centers.
L'archive ouverte pluridisciplinaire HAL, est destinée au dépôt et à la diffusion de documents scientifiques de niveau recherche, publiés ou non, émanant des établissements d'enseignement et de recherche français ou étrangers, des laboratoires publics ou privés. 


\title{
Domain Decomposition Methods and High-Order Absorbing Boundary Conditions for the Numerical Simulation of the Time Dependent Schrödinger Equation with Ionization and Recombination by Intense Electric Field
}

\author{
X. Antoine ${ }^{\mathrm{a}, \mathrm{b}}$, E. Lorin ${ }^{\mathrm{d}, \mathrm{c}}$, A. D. Bandrauk $\mathrm{e}^{\mathrm{e}, \mathrm{c}}$ \\ ${ }^{a}$ Institut Elie Cartan de Lorraine, Université de Lorraine, F-54506 Vandoeuvre-lès-Nancy Cedex, France \\ ${ }^{b}$ Inria Nancy Grand-Est/IECL - ALICE. \\ ${ }^{c}$ Centre de Recherches Mathématiques, Université de Montréal, Montréal, Canada, H3T 1J4 \\ ${ }^{d}$ School of Mathematics and Statistics, Carleton University, Ottawa, Canada, K1S 5B6 \\ ${ }^{e}$ Laboratoire de Chimie Théorique, Université de Sherbrooke, Sherbrooke, Canada, J1K 2R1
}

\begin{abstract}
This paper is devoted to the efficient computation of the Time Dependent Schrödinger Equation (TDSE) for quantum particles subject to intense electromagnetic fields including ionization and recombination of electrons with their parent ion. The proposed approach is based on a domain decomposition technique, allowing a fine computation of the wavefunction in the vicinity of the nuclei located in a domain $\Omega_{1}$ and a fast computation in a roughly meshed domain $\Omega_{2}$ far from the nuclei where the electrons are assumed free. The key ingredients in the method are i) well designed transmission boundary conditions on $\partial \Omega_{1}$ (resp. $\partial \Omega_{2}$ ) in order to estimate the part of the wavefunction "leaving" Domain $\Omega_{1}$ (resp. $\Omega_{2}$ ), ii) a Schwarz waveform relaxation algorithm to accurately reconstruct the solution. The developed method makes it possible for electrons to travel from one domain to another without loosing accuracy, when the frontier or the overlapping region between two domains is crossed by the wavefunction.
\end{abstract}

Keywords: Schrödinger equation, non-reflecting boundary conditions, domain decomposition method, Schwarz waveform relaxation, laser, ionization

\section{Introduction}

The goal of this paper is to provide an efficient, easy-to-implement and accurate numerical approach for solving the Time Dependent Schrödinger Equation (TDSE), [16], [13], [17], [11] when molecules are subject to intense laser pulses. The method is based on a domain decomposition technique and the derivation of accurate non-reflecting boundary conditions used as quasi-optimal transmission boundary conditions between the subdomains. Subject to intense and short laser pulses, an electron is first extracted from its parent ion, is then "freed", and finally goes back in the vicinity of its nucleus [28]. Of main interests are highly nonlinear phenomena such as High Harmonic Generation (HHG), or Above Threshold Ionization (ATI), [27], [32] which occur in the vicinity of the nuclei and which are caused by the recombination of high energy electrons with their parent ion. This process is repeated for each intense enough laser pulse cycle. From a numerical point of view, the ionization necessitates the use of an extended computational domain, and as a consequence important computational resources [2], [26] as several time and space scales are involved in this problem. As any other wave-like problem, the derivation and approximation of non-reflecting boundary conditions is a major question, which was treated extensively in the literature, from empirical absorbing boundary conditions (ABC) [12, 2], [14], Perfectly Matched Layers (PMLs) [15, 3, 2], as well as microlocal analysis methods starting from the pioneering work by Engquist and Majda [18], then Halpern and Rauch [23] for

Email addresses: xavier.antoine@univ-lorraine.fr (X. Antoine), elorin@math.carleton.ca (E. Lorin), andre.bandrauk@usherbrooke.ca (A. D. Bandrauk) 
the diffusion equation, and more recently by Antoine, Besse [7], [6], [4] and Szeftel [10], [34] for the linear and nonlinear Schrödinger equations by using pseudo- and paradifferential operator techniques. We refer to [2] for a full literature review on absorbing boundary conditions for TDSE. The goal of the present paper is not to derive new absorbing boundary conditions for the TDSE in order to avoid or limit reflections at domain boundary. We are here interested in the global solution of the laser-molecule TDSE including the ionization and recombination processes. In this goal non-reflecting boundary conditions are not sufficient but will constitute one of the key ingredients, for which the chosen approach is one of the most accurate, and which was developed in [7]. Note that any other ABC could in principle be used [31] for instance. The other key ingredient is the domain decomposition algorithm to connect the solution in each subdomain. The chosen method is the optimized Schwarz waveform relaxation algorithm (OSWR) [21], [19], [20] and which was originally proposed in [24] for the TDSE. In the OSWR method for domain decomposition, transparent boundary conditions are used to derive transmission conditions between subdomains. Using nonlocal transmission conditions was suggested earlier, in particular, in a paper [22] on domain decomposition for nonlinear elliptic boundary problems.

In this paper, we focus on one single electron of an atomic system, but the principle of the method is still valid and in fact relevant for several electron problems. More specifically, the problem which is considered in this paper is as follows. We assume that the electron is at the ground state $u_{0}$ of energy $E_{0}$ of the laser-free Hamiltonian $H_{0}=-\triangle+V_{c}$, that is

$$
H_{0} u_{0}=E_{0} u_{0}
$$

Function $V_{c}$ is the interaction potential depending on the considered particle. The electron is then subject to a laser field $\mathbf{E}$ which will be assumed to be time-dependent only, which constitutes a reasonable assumption at the molecule or atom scale (dipole approximation) [29]. The equation writes, in the so-called length gauge, for all $(\mathbf{r}, t) \in \mathbb{R}^{3} \times[0, T]$ :

$$
i \partial_{t} u(\mathbf{r}, t)=-\triangle u(\mathbf{r}, t)+V_{c}(\mathbf{r}) u(\mathbf{r}, t)+\mathbf{r} \cdot \mathbf{E}(t) u(\mathbf{r}, t)
$$

Note that a simple gauge transform [13], leads to the new form, the so called velocity gauge

$$
i \partial_{t} u(\mathbf{r}, t)=-\triangle u(\mathbf{r}, t)+V_{c}(\mathbf{r}) u-i \mathbf{A}(t) \cdot \nabla u(\mathbf{r}, t)+\|\mathbf{A}\|^{2} u(\mathbf{r}, t)
$$

where this time the laser operator appears as the sum of a differential and algebraic operators, and where $\mathbf{A}$ is the electric potential, which is an anti-derivative of the electric field. The derived method is independent of the chosen gauge, both gauges presented above could be used. The velocity gauge will be here more convenient for the simplified method which will be proposed in Section 2.3. In the following, we will omit the variables $(\mathbf{r}, t)$ in the equations. In this paper, we also assume that the electric field is a regular traveling wave propagating at the speed of light, $c$. In practice, a truncation of the overall domain is usually necessary, as well as boundary conditions on $\partial \Omega$. In order to include ionization in the simulation, $\Omega$ should be chosen very large, which is in practice numerically very expensive. In addition:

- the interaction potential is singular (or quasi-singular), which necessitates a fine resolution near the nuclei.

- efficient TDSE numerical solvers (finite element or implicit finite difference schemes) are usually computationally complex, as they necessitate the numerical computation of sparse linear system solutions.

- wavefunction solution to the TDSE are nonlocal.

- this problem is multiscale, due to complex phenomena such as for instance, high harmonic generation and multiphoton-ionization, that is the generation of photons of frequency that could reach hundred times the frequency of the incoming pulse. As a consequence, even for simple molecules the interaction with an intense laser pulse can generate scales in time, from attosecond to picosecond scales, and in space from picometers to tens of nanometers, see for instance [28], [32], [27], [30] and [13] for a more details. 
A possible approach, to partially circumvent this problem consists of using an adaptive or not, non-uniform mesh: fine close to the nucleus, and coarse in the ionization zone. Although very attracting, this approach has also major issues: stable and accurate numerical solvers are based on implicit techniques, requiring the numerical solution of large and potentially ill-conditioned linear systems, slowing down the convergence of iterative linear system solvers. In addition, at each iteration, the solution at one point in space is a priori dependent of the solution in all the computational domain. The use of large cells may then deteriorate the solution everywhere, in particular in fine resolution regions. Domain decomposition method is another class of numerical techniques for solving complex and high dimensional problems. The principle of the method which is used here is now described. First, the bounded, open and convex domain $\Omega \subseteq \mathbb{R}^{d}$ is decomposed in two regions $\Omega_{1,2}$ such that $\Omega=\Omega_{1} \cup \Omega_{2}$ and where $\mathcal{E}$ denotes the nonempty overlapping domain $\Omega_{1} \cap \Omega_{2}$. In addition we assume that $\mathcal{E}$ have smooth boundary, and that $\Omega_{1}-\mathcal{E}$ contains the supports of $u_{0}$. In $\Omega_{1}$, we approximate TDSE using a fine mesh. In $\Omega_{2}$ a rough mesh may be used. TDSE will be solved "independently" in domains $\Omega_{1,2}$, allowing a reduction of the overall computational complexity. The region $\mathcal{E}$ is supposed to be of infinitely small thickness. The size of the overlaping domain is one of the most important criteria

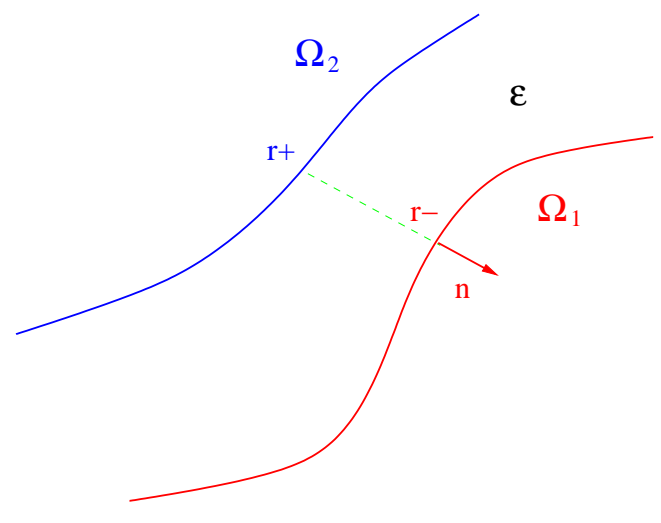

Figure 1: Thickness at $\mathbf{r}^{-} \in \Omega_{1}$

in domain decomposition methods, in terms of rate of convergence and computational complexity. The ideal case being a rapid convergence without overlaping. Following ideas from [24], we can expect that the convergence rate will be strongly dependent on the frequency, the size and the shape of the overlapping region, as well as the interaction potential and the electric field. In addition, although analytically, the transmission conditions can be derived, at least formally, from transparent boundary conditions, they are in practice implemented using $\mathrm{ABC}$. The rate of convergence of the fixed point algorithm will then also be dependent on the order of approximation of the ABC.

In order to connect the solution in each domain, appropriate boundary conditions on $\partial \Omega_{1}$ and $\partial \Omega_{2}$ have then to be derived as well as a fixed point algorithm. This is the object of this paper. In the following, we will denote by $\Sigma$ the external boundary of $\Omega$ (and of $\Omega_{2}$ ), $\Gamma^{+}$the internal boundary of $\Omega_{2}$ and $\Gamma^{-}$the (external) boundary of $\Omega_{1}$. In practice and from there, we will assume that both $\Omega_{1}, \Omega$ are convex and $\Omega_{2}$ is concave(interior)-convex(exterior). Note that the thickness of $\mathcal{E}$ is assumed infinitely small $\mathbf{n}_{\Gamma^{-}} \sim-\mathbf{n}_{\Gamma^{+}}$. In practice, $\Omega_{2}$ will cover a large external region which will be meshed with large cells. The solution transmission from $\Omega_{1}$ to $\Omega_{2}$ will be done using two main ingredients i) pseudodifferential transparent/absorbing boundary conditions and ii) the quasi-optimal Schwarz waveform relaxation algorithm [24] which is a sophisticated fixed point method.

In the following $H^{(i)}$ stands for the Schrödinger Hamiltonian (in length or velocity gauge) in Domain $\Omega_{i}$, $i=1,2$ and $\mathcal{B}_{\Gamma^{ \pm}}$the corresponding boundary operator. From now, we assume that $V_{c}$ has its support in $\Omega_{1}$. Note that it is not a mathematical constraint of the method, and remark that the overall potential $V_{c}+\mathbf{x} \cdot \mathbf{E}(t)$ has not compact support in $\Omega_{1}$. 
In $\Omega_{2}$, the equation writes for all $k \geqslant 1$ :

$$
\left\{\begin{array}{l}
i \partial_{t} u_{2}^{(k)}=H^{(2)} u_{2}^{(k)} \text { in } \Omega_{2} \\
\mathcal{B}_{\Gamma^{+}} u_{2}^{(k)}=\mathcal{B}_{\Gamma^{+}} u_{1}^{(k-1)} \text { on } \Gamma^{+} \\
\mathcal{B}_{\Sigma} u_{2}^{(k)}=0 \text { on } \Sigma \\
u_{2}^{(k)}(\cdot, 0)=0
\end{array}\right.
$$

where in velocity gauge the Hamiltonian writes for $\mathbf{r} \in \Omega_{2}$ :

$$
H_{v}^{(2)}(t)=-\triangle-i \mathbf{A}(t) \cdot \nabla+\|\mathbf{A}(t)\|^{2}
$$

and

$$
H_{l}^{(2)}(\mathbf{r}, t)=-\triangle-i \mathbf{r} \cdot \mathbf{E}(t)
$$

in length gauge. In addition, $\mathcal{B}_{\Gamma^{ \pm}}, \mathcal{B}_{\Sigma}$ are operators to determine and with an initial condition $u_{2}$ chosen identically null. In $\Omega_{1}$, with $\Gamma^{+} \subseteq \Omega_{1}$, the equation writes:

$$
\left\{\begin{array}{l}
i \partial_{t} u_{1}^{(k)}=H^{(1)} u_{1}^{(k)} \text { in } \Omega_{1}, \\
\mathcal{B}_{\Gamma^{-}} u_{1}^{(k)}=\mathcal{B}_{\Gamma^{-}} u_{2}^{(k-1)} \text { on } \Gamma^{-}, \\
u_{1}^{(k)}(\cdot, 0)=u_{0}(\cdot),
\end{array}\right.
$$

where in velocity gauge the Hamiltonian writes for $\mathbf{r} \in \Omega_{1}$ :

$$
H_{v}^{(1)}(t)=-\triangle+V_{c}(\mathbf{r})-i \mathbf{A}(t) \cdot \nabla+\|\mathbf{A}(t)\|^{2}
$$

and

$$
H_{l}^{(1)}(\mathbf{r}, t)=-\triangle+V_{c}(\mathbf{r})-i \mathbf{r} \cdot \mathbf{E}(t) .
$$

The boundary conditions will be derived to formally obtain a pointwise convergence almost everywhere for all $t \geqslant 0, u_{1}^{(k)} \rightarrow_{k \rightarrow+\infty} u_{\mid \Omega_{1}}$ and $u_{2}^{(k)} \rightarrow_{k \rightarrow+\infty} u_{\mid \Omega_{2}}$. In order to initiate the iterations, it is necessary to impose initial guess $\mathcal{B}_{\Gamma^{-}} u_{1}^{(0)}=g_{1}, \mathcal{B}_{\Gamma^{+}} u_{2}^{(0)}=g_{2}$. Note that it was proven in [24] that using transparent boundary conditions (Dirichlet-to-Neumann (DtN) maps), this procedure is convergent in 2 iterations for every initial guess $\left(g_{1}, g_{2}\right)$ in the free one-dimensional case. More generally, and this is true in our situation, an approximate DtN operator is rather used, slowing down the convergence. Boundary condition $\mathcal{B}_{\Sigma}$ on $\Sigma$ is chosen to avoid spurious wave reflections. There is no particular difficulty here as we do not require any information outside $\Omega$. We use the absorbing conditions, skipping the details that can be found for instance in [5]. In the following, as the transmission conditions will be derived from DtN-like operators, $\mathcal{B}$ will be searched in the form $\partial_{\mathbf{n}}+B$, where $\mathbf{n}$ is a normal vector to the considered boundary, and where $B$ is an operator to determine.

The rest of the paper is organized as follows. Section 2 is devoted to the derivation and some analysis of the proposed domain decomposition technique. Section 3 is devoted to the approximation of the derived method, and numerical results are presented in Section 4 . We finally conclude in Section 5.

\section{Solution reconstruction}

The general principle for deriving transmission conditions is as follows. On $\Omega_{1}$ and $\Omega_{2}$ the boundary conditions are first constructed, to be transparent or absorbing, using some techniques which will allow us to accurately separate incoming from outgoing waves. In a second stage, the outgoing waves from $\Omega_{1}$ have to be "transmitted" to $\Omega_{2}$ through the overlapping domain $\mathcal{E}$, and similarly any outgoing wave from $\Omega_{2}$ going towards $\Omega_{1}$ has to be "transmitted" to $\Omega_{1}$. 


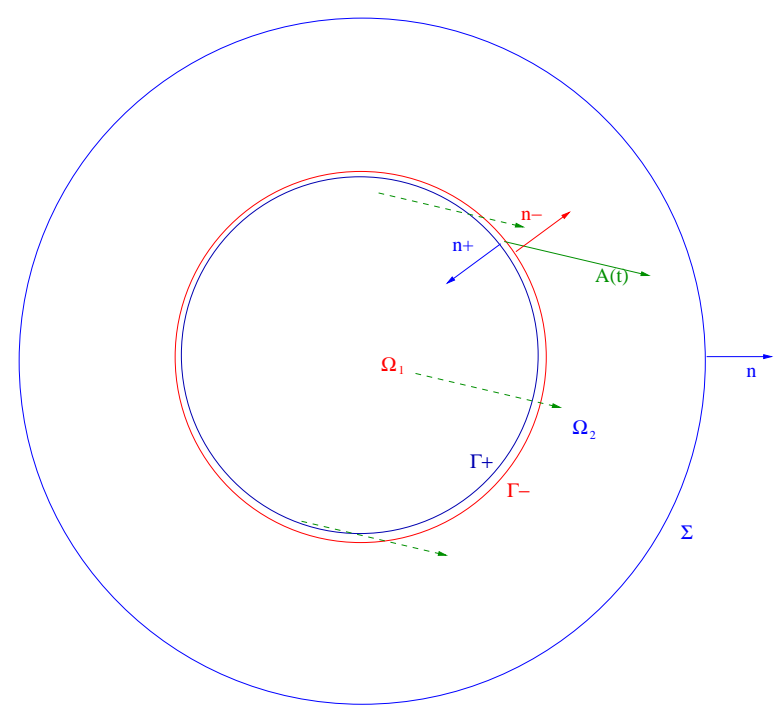

Figure 2: Geometric configuration

\subsection{Absorbing, incoming and outgoing boundary conditions}

The general solution to

$$
i \partial_{t} u(\mathbf{r}, t)=H u(\mathbf{r}, t)
$$

with $H=H_{v}$ (resp. $H=H_{l}$ ) is given by (5) (resp. (6)), is constructed, in the ideal case, as follows

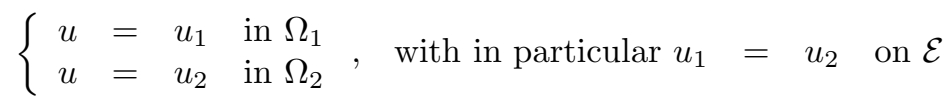

For all $k \geqslant 1$ and for all $t \in[0, T]$, we first solve the equation in $\Omega_{1,2}$ according to [24], (4), (3). From a practical point of view, the construction of the explicit boundary conditions is far from trivial. They are constructed by factorizing the Schrödinger Hamiltonian in the hyperbolic region of the cotangent bundle of the domain boundary, then by identifying the incoming and outgoing waves. The later step is possible by analyzing the null bicharacteristic strip solutions of a set of Hamiltonian equations [25], [1].

- Boundary condition on $\Sigma$. The chosen absorbing boundary condition on $\Omega_{2}$ is based on a method which was developed in [5] and [7]. These boundary conditions are non-reflecting and are of the form:

$$
\text { for all } \mathbf{r} \in \Sigma \text { and for all } t \geqslant 0:\left(\partial_{\mathbf{n}}+B_{\Sigma}\right) u_{2}^{(k)}=0
$$

with $\mathbf{n}$ the outward normal vector at $\mathbf{r} \in \Sigma$, and where $\partial_{\mathbf{n}}+B_{\Sigma}$ is associated to outgoing waves from $\Omega_{2}$ through $\Sigma$. Recall that at this stage, any other accurate $A B C$ could a priori be used.

- Boundary condition on $\Gamma^{-}$. The goal is to develop boundary conditions at any point $\mathbf{r}^{-} \in \Gamma^{-}$and all time $t$ which are non-reflecting for outgoing waves from $\Omega_{1}$, and allowing incoming from $\Omega_{2}$ to enter and propagate in $\Omega_{1}$. In $\Omega_{1}$, we have

$$
i \partial_{t} u_{1}^{(k)}=H^{(1)} u_{1}^{(k)}
$$

and

$$
\text { for all } \mathbf{r}^{-} \in \Gamma^{-} \text {and for all } t \geqslant 0:\left(\partial_{\mathbf{n}^{-}}+B_{\Gamma^{-}}^{+}\right) u_{1}^{(k)}=\left(\partial_{\mathbf{n}^{-}}+B_{\Gamma^{-}}^{+}\right) u_{2}^{(k-1)}
$$

with $\mathbf{n}^{-}$the outward normal vector at $\mathbf{r}^{-} \in \Gamma^{-}$, where $\partial_{\mathbf{n}^{-}}+B_{\Gamma^{-}}^{+}$characterizes the outgoing waves from $\Omega_{1}$. 
- Boundary condition on $\Gamma^{+}$. Similarly for $\mathbf{r} \in \Gamma^{+}$

$$
i \partial_{t} u_{2}^{(k)}=H^{(2)} u_{2}^{(k)}
$$

in $\Omega_{2}$ and the boundary condition writes:

$$
\text { for all } \mathbf{r}^{+} \in \Gamma^{+} \text {and for all } t \geqslant 0:\left(\partial_{\mathbf{n}^{+}}+B_{\Gamma^{+}}^{+}\right) u_{2}^{(k)}=\left(\partial_{\mathbf{n}^{+}}+B_{\Gamma^{+}}^{+}\right) u_{1}^{(k-1)}
$$

with $\mathbf{n}^{+}$outward normal vector at $\mathbf{r} \in \Gamma^{+}$, and where $\partial_{\mathbf{n}^{+}}+B_{\Gamma^{+}}^{+}$is associated to outgoing waves from $\Omega_{2}$.

\subsection{Construction of the global solution in any dimension}

We present in this subsection the reconstruction algorithm based on absorbing boundary conditions and Schwarz waveform relaxation method, [24], [19], [20].

Proposition 2.1. For $t \in\left[t_{a}, t_{b}\right]$ and with $t_{b}=t_{a}+\delta$ and $w_{1,2}$ the initial data at $t=t_{a}$ in $\Omega_{1,2}$ (computed from previous times), we solve for $k \geqslant 1$

1. in $\Omega_{1}$, and $t \in\left[t_{a}, t_{b}\right)$

$$
\begin{cases}i \partial_{t} u_{1}^{(k)} & =H^{(1)} u_{1}^{(k)} \text { in } \Omega_{1}, \\ \left(\partial_{\mathbf{n}^{-}}+B_{\Gamma^{-}}^{+}\right) u_{1}^{(k)} & =\left(\partial_{\mathbf{n}^{-}}+B_{\Gamma^{-}}^{+}\right) u_{2}^{(k-1)} \text { on } \Gamma^{-}, \\ u_{1}\left(\cdot, t_{a}\right) & =u_{0}(\cdot) .\end{cases}
$$

2. in $\Omega_{2}\left(V_{c}(\mathbf{r})=0\right.$ for $\left.\mathbf{r} \in \Omega_{2}\right)$, and $t \in\left[t_{a}, t_{b}\right)$

$$
\begin{cases}i \partial_{t} u_{2}^{(k)} & =H^{(2)} u_{2}^{(k)} \text { in } \Omega_{2}, \\ \left(\partial_{\mathbf{n}^{+}}+B_{\Gamma^{+}}^{+}\right) u_{2}^{(k)} & =\left(\partial_{\mathbf{n}^{+}}+B_{\Gamma^{+}}^{+}\right) u_{1}^{(k-1)} \text { on } \Gamma^{+}, \\ \left(\partial_{\mathbf{n}}+B_{\Sigma}\right) u_{2}^{(k)} & =0 \text { on } \Sigma, \\ u_{2}^{(k)}\left(\cdot, t_{a}\right) & =0,\end{cases}
$$

with an imposed initial guess $\left(u_{1}^{(0)}, u_{2}^{(0)}\right)$.

Remark 2.1. An alternative method to the one presented in Prop. 2.1 is as follows.

1. in $\Omega_{1}$, boundary conditions are changed into:

$$
\left(\partial_{\mathbf{n}^{-}}+B_{\Gamma^{-}}^{+}\right) u_{1}^{(k)}=0 \text { on } \Gamma^{-}
$$

2. in $\Omega_{2}$, boundary conditions on $\Gamma^{+}$are changed into:

$$
\left(\partial_{\mathbf{n}^{+}}+B_{\Gamma^{+}}^{+}\right) u_{2}^{(k)}=0 \text { on } \Gamma^{+}
$$

3. Finally, at time $t_{b}$ we add the conditions:

$$
\left\{\begin{array}{l}
u_{1}^{(k)}\left(\mathbf{r}^{-}, t_{b}\right)=u_{2}^{(k-1)}\left(\mathbf{r}^{+}, t_{b}\right), \\
u_{2}^{(k-1)}\left(\mathbf{r}^{+}, t_{b}\right)=u_{1}^{(k-1)}\left(\mathbf{r}^{-}, t_{b}\right)
\end{array}\right.
$$


Note that as $\left[\triangle, \mathbf{A} \cdot \nabla+\|\mathbf{A}(t)\|^{2}\right]=0$, we can split the equation into $i \partial_{t} u_{2}=-i \mathbf{A}(t) \cdot \nabla u_{2}+\|\mathbf{A}(t)\|^{2} u_{2}$ and $i \partial_{t} u_{2}(\mathbf{r}, t)=-\triangle u_{2}(\mathbf{r}, t)$. The transport equation has a simple analytical solution. Indeed, assuming that $w=u_{2}\left(\cdot, t_{a}\right)$ is known at time $t_{a}$, then the solution at time $t_{b}>t_{a}$ it is of the form

$$
u_{2}\left(\cdot, t_{b}\right)=w_{2}\left(\cdot-\int_{t_{a}}^{t_{b}} \mathbf{A}(s) d s\right) e^{-i \int_{t_{a}}^{t_{b}}\|\mathbf{A}(s)\|^{2} d s}
$$

Note that in length gauge, this commutation property would not be satisfied.

The choice of these boundary conditions is justified by:

- boundary conditions at $\Gamma^{ \pm}$are designed for outgoing waves, from $\Omega_{1}$ (resp. $\Omega_{2}$ ) to $\Omega_{2}$ (resp. $\Omega_{1}$ ).

- the equation is linear and as consequence two waves propagating in opposite directions will not nonlinearly "interfere".

Note that the approach which was presented above is totally independent of the choice of the system of coordinates as well as the choice of $\mathrm{ABC}$, provided they are accurate enough.

The method which is presented here can in principle be quite inefficient if the convergence is slow. This is precisely why "quasi-optimal" high-order transmission boundary conditions should be used. We now state some basic results about convergence and simple ways to improve the overall computational complexity of the method.

Proposition 2.2. Assume that $\Omega=\cup_{i=1}^{m} \Omega_{i}$ is decomposed into $m$ subdomains $\Omega_{i}$. For all $k \in\{2, \cdots, m-1\}$ assume that i) $l \notin\{k, k-1, k+1\}$ implies $\Omega_{l} \cap \Omega_{k}=\emptyset$ and ii) $l \in\{k-1, k+1\}$ implies $\Omega_{l} \cap \Omega_{k}$ is an overlapping interface as in Fig. 3. Let $u_{i}$ be the corresponding solution on $\Omega_{i}$. With this configuration, if at Schwarz iteration $k \geqslant 1$, we have

- $u_{0 \mid \Omega_{i}}=0$ for all $i>1$,

- $u_{i}^{(k-1)}=u_{i-1}^{(k-1)}=u_{i+1}^{(k-1)}=0$

then $u_{i}^{(k)}=0$, so that computation in $\Omega_{i}$ at iteration $k$ is unnecessary.

Proof. The proof is straightforward as at iteration $k$, the condition imposed at $\partial \Omega_{i}$ involves $u_{i}^{(k)}, u_{i-1}^{(k-1)}$ and $u_{i+1}^{(k-1)}$.

This proposition although simple has important consequences from a practical point of view, as it al-

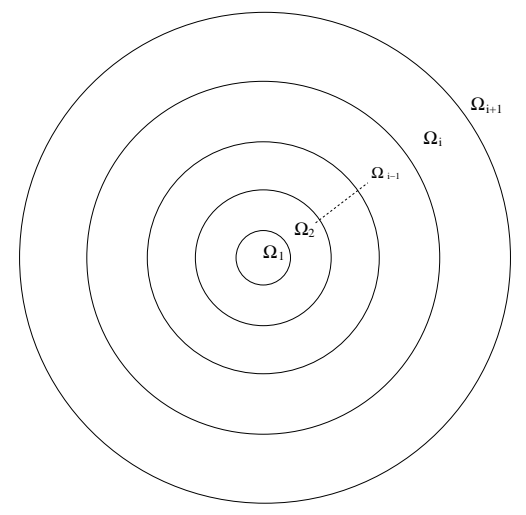

Figure 3: Geometric configuration - Prop. 2.2, 2.3

lows to reduce (and in fact to avoid) unnecessary computations. In particular 
Corollary 2.1. Assuming that $\Omega$ is decomposed as in Prop. 2.2, and that ii) $u_{0}$ is null except in $\Omega_{1}$, then at iteration $k \leqslant m-2, u_{l}^{(k)}=0$ for all $l \geqslant k+2$, any computation in $\left(\Omega_{l}\right)_{l \geqslant k+2}$ is unnecessary.

On a broader picture, the overall convergence of the Schwarz process is reached when convergence is reached in each overlapping domains. Similarly to the above proposition, we can state an interesting, although more restrictive proposition.

Proposition 2.3. Under the same assumptions as Prop. 2.2 and assuming also that for each overlapping domain $\Omega_{i} \cap \Omega_{i+1}, i \in\{1, \cdots, m\}$, convergence is reached in $p$ iterations (quasi-optimal convergence for $p=2)$, then

- for $k \in\{p, 2 p, \cdots, p m\}$ and any $l \geqslant 1$, we have $u_{i}^{(k+l)}=u_{i}^{(k)}$, for all $i \in\{1,2, \cdots, k / p\}$,

- the overall convergence is reached in at most $p(m-1)$ iterations,

- each Schwarz iteration will require, at least TDSE computations in p subdomains.

Proof. From to the hypothesis about convergence in $p$ iterations, we easily deduce by induction that at iteration $k \in\{p, 2 p, \cdots, p m\}$, convergence is reached in overlapping domain $\Omega_{i} \cap \Omega_{i+1}$, for all $i \in\{1, \cdots, k / p\}$, from which we deduce the first statement. As a consequence, the overall convergence will be reached after $p(m-1)$ Schwarz iterations. Finally from Schwarz iteration $k \in\{p, 2 p, \cdots, p m\}$, any computation in domains $\Omega_{i}$ with $i \in\{1, \cdots, k / p\}$ becomes unnecessary as convergence has been reached in these domains.

Again we take advantage of this result to limit the overall computational complexity. Note however that the hypothesis on the convergence in each overlapping domain is a priori not trivially satisfied and may be complicated to justify in practice.

\subsection{Simplified solution reconstruction with no explicit numerical computation in $\Omega_{2}$}

In this section, only, we assume that the overlapping domain $\mathcal{E}=\Omega \cap \Omega_{2}$ is given by $\mathcal{E}=\Gamma^{-}=\Gamma^{+}$. We discuss a simplified version of the method which was developed in Section 2.2. The main issue in Section 2.2 comes from the fact that it is required to solve a TDSE in $\Omega_{2}$, which increases the overall computational complexity of the method. In addition the Schwarz algorithm is not necessary in this simplified approach. In order to avoid this issue, a simple idea consists of neglecting in $\Omega_{2}$ the effect of the kinetic operator and considering the electron as a classical particle. This is naturally not physical. Recall, however that i) we are not interested in the solution inside $\Omega_{2}-\Omega_{1}$, but only in $\Omega_{1}$ and ii) the $L^{2}-$ norm of the wavefunction in $\Omega_{2}$ (then in $\mathbb{R}^{3}$ ) will still be conserved. Indeed, in that case, the electron is only driven by the electric field and as a consequence, we can determine analytically the particle trajectory in $\Omega_{2}$, see Fig. 4 . We do not expect that the overall will converge to the exact solution in all $\mathbb{R}^{3}$, but rather an efficient computation to a sufficiently accurate solution in $\Omega_{1}$. We take advantage to the fact that $\mathbf{A}$ is time-dependent only and then to the fact that the following IBVP, for $t \in[0, T]$

$$
\begin{cases}i \partial_{t} u_{2} & =-i \mathbf{A}(t) \cdot \nabla u_{2}+\|\mathbf{A}(t)\|^{2} u_{2} \text { in } \Omega_{2}, \\ u_{2} & =w \text { if } \mathbf{A}(t) \cdot \mathbf{n}<0 \text { on } \Gamma^{+} \\ u_{2}(\cdot, 0) & =w_{2}(\cdot)\end{cases}
$$

has an explicit solution. Assume first that at time $t_{0}$ and in $\mathbf{r}_{\alpha}^{+} \in \Gamma^{+}, \mathbf{A}\left(t_{0}\right) \cdot \mathbf{n}_{\alpha}<0$. That is $u_{2}\left(\mathbf{r}_{\alpha}^{+}, t_{0}\right)=$ $w\left(\mathbf{r}_{\alpha}^{+}, t_{0}\right)$, where $w$ is a given function. In practice $w$ will given by $u_{1}$ on $\Gamma^{-}$. Assume in addition that at time $t^{*}>t_{0}, \mathbf{r}_{\alpha}^{+}+\int_{t_{0}}^{t^{*}} \mathbf{A}(s) d s=\mathbf{r}_{\beta}^{+}$for some $\mathbf{r}_{\beta}^{+} \in \Gamma^{+}$. In that case, we easily deduce that

$$
u_{2}\left(\mathbf{r}_{\beta}^{+}, t^{*}\right)=w\left(\mathbf{r}_{\alpha}^{+}, t_{0}\right) e^{-i \int_{t_{0}}^{t^{*}}\|\mathbf{A}(s)\|^{2} d s}
$$

In the framework of our domain decomposition approach, this quantity would be taken, neglecting kinetic effects in $\Omega_{2}$, such that

$$
u_{1}\left(\mathbf{r}_{\beta}^{-}, t^{*}\right)=u_{2}\left(\mathbf{r}_{\beta}^{+}, t^{*}\right)=w\left(\mathbf{r}_{\alpha}^{+}, t_{0}\right) e^{-i \int_{t_{0}}^{t^{*}}\|\mathbf{A}(s)\|^{2} d s}
$$




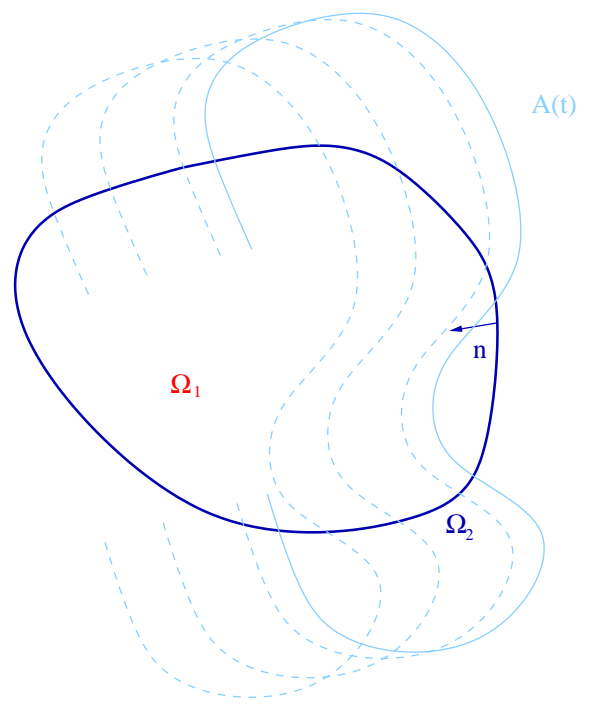

Figure 4: Electric potential

Inversely, assume that at time $t^{*}$ and in $\mathbf{r}_{\alpha}^{+} \in \Gamma^{+}, \mathbf{A}\left(t^{*}\right) \cdot \mathbf{n}_{\alpha}>0$, then 2 cases can occur:

- Assume that for all $t \in\left[0, t^{*}\right)$

$$
\mathbf{r}_{\alpha}^{+}-\int_{0}^{t^{*}} \mathbf{A}(s) d s \notin \Gamma^{+}
$$

then

$$
u_{2}\left(\mathbf{r}_{\alpha}^{+}, t^{*}\right)=w_{2}\left(\mathbf{r}_{\alpha}^{+}-\int_{t_{0}}^{t^{*}} \mathbf{A}(s) d s\right) e^{-i \int_{t_{0}}^{t^{*}}\|\mathbf{A}(s)\|^{2} d s}
$$

Note that in practice, we will choose $w_{2}=0($ at $t=0)$, as we have assumed initially that the support of the initial data is included in $\Omega_{1}$.

- Assume that there exists $t_{i} \in\left[0, t^{*}\right)$ and $\mathbf{r}_{\beta_{i}}^{+} \in \Gamma^{+}$with

$$
\mathbf{r}_{\beta_{i}}^{+}=\mathbf{r}_{\alpha}^{+}-\int_{t_{i}}^{t^{*}} \mathbf{A}(s) d s
$$

Recall that the internal boundary of $\Omega_{2}$ is concave and $\mathbf{A}$ space-independent, then $\mathbf{r}_{\beta_{i}}^{+}$is of course unique. However $t_{i}$ is not necessarily unique. So we denote:

$$
\bar{t}:=\sup \left\{t_{i} \in\left[0, t^{*}\right), \text { s.t. } \exists \mathbf{r}_{\beta_{i}}^{+} \in \Gamma^{+}, \text {with } \mathbf{r}_{\alpha}^{+}-\int_{t_{i}}^{t^{*}} \mathbf{A}(s) d s=\mathbf{r}_{\beta_{i}}^{+}\right\}
$$

We also denote by $\overline{\mathbf{r}}_{\beta}^{+}$and $\overline{\mathbf{n}}_{\beta}$ the corresponding $\mathbf{r}_{\beta}^{+}$and outward normal vector at $\overline{\mathbf{r}}_{\beta}^{+}$. Then we conclude that the value at time $t^{*}$, and in $\mathbf{r}_{\alpha}^{+}$is given by:

$$
u_{2}\left(\mathbf{r}_{\alpha}^{+}, t^{*}\right)=u_{2}\left(\overline{\mathbf{r}}_{\beta}^{+}, \bar{t}\right) e^{-i \int_{\bar{t}}^{t^{*}}\|\mathbf{A}(s)\|^{2} d s}=u_{2}\left(\mathbf{r}_{\alpha}^{+}-\int_{\bar{t}}^{t^{*}} \mathbf{A}(s) d s, \bar{t}\right) e^{-i \int_{\bar{t}}^{t^{*}}\|\mathbf{A}(s)\|^{2} d s}
$$

which is in fact

$$
u_{2}\left(\mathbf{r}_{\alpha}^{+}, t^{*}\right)=w\left(\overline{\mathbf{r}}_{\beta}^{+}, \bar{t}\right) e^{-i \int_{\bar{t}}^{t^{*}}\|\mathbf{A}(s)\|^{2} d s}=w\left(\mathbf{r}_{\alpha}^{+}-\int_{\bar{t}}^{t^{*}} \mathbf{A}(s) d s, \bar{t}\right) e^{-i \int_{\bar{t}}^{t^{*}}\|\mathbf{A}(s)\|^{2} d s}
$$


as necessarily in $\overline{\mathbf{r}}_{\beta}^{+}, \mathbf{A}(\bar{t}) \cdot \overline{\mathbf{n}}_{\beta} \geqslant 0$. Indeed, if $\mathbf{A}(\bar{t}) \cdot \overline{\mathbf{n}}_{\beta}$ was negative, there would exist a $\tilde{t}>\bar{t}$, such that $\mathbf{r}_{\alpha}^{+}-\int_{\tilde{t}}^{t^{*}} \mathbf{A}(s) d s=\overline{\mathbf{r}}_{\beta}^{+}$and $\mathbf{A}(\tilde{t}) \cdot \overline{\mathbf{n}}_{\beta} \geqslant 0$, which is in contradiction with the definition of $\bar{t}$.

In practice, in order to implement these boundary conditions, at time $t^{*}$, we have to integrate $\mathbf{A}$ from time $t^{*}$ to $t$ such that $\mathbf{r}_{\alpha}^{+}-\int_{t}^{t *} \mathbf{A}(s) d s \in \Gamma^{+}$.

The overall reconstruction of the solution in $\Omega$ is summarized in the following proposition.

Proposition 2.4. For $t \in\left(t_{a}, t_{a}+\delta\right)$, we define $t_{b}=t_{a}+\delta$ and $w_{1,2}$ the initial data at $t=t_{a}$, in $\Omega_{1,2}$ (computed from previous times), where $\Omega_{2}$ is the complementary in $\mathbb{R}^{3}$ of $\Omega_{1}$. For $t \in\left(t_{a}, t_{b}\right)$, we solve

1. in $\Omega_{1}$

$$
\begin{cases}i \partial_{t} u_{1} & =-\Delta u_{1}+V_{c}(\mathbf{r}) u_{1}-i \mathbf{A}(t) \cdot \nabla u_{1}+\|\mathbf{A}(t)\|^{2} u_{1} \text { in } \Omega_{1}, \\ \left(\partial_{\mathbf{n}^{-}}+B_{\Gamma^{-}}^{+}\right) u_{1} & =0 \text { on } \Gamma^{-}, \\ u_{1}\left(\cdot, t_{a}\right) & =w_{1}(\cdot)\end{cases}
$$

2. in $\Omega_{2}\left(V_{c}(\mathbf{r})=0\right.$, for $\left.\mathbf{r} \in \Omega_{2}\right)$

- if for all $t \in\left[t_{a}, t_{b}\right)$

$$
\mathbf{r}_{\alpha}^{+}-\int_{t_{a}}^{t_{b}} \mathbf{A}(s) d s \notin \Gamma^{+}
$$

then

$$
u_{2}\left(\mathbf{r}_{\alpha}^{+}, t_{b}\right)=u_{2}\left(\mathbf{r}_{\alpha}^{+}-\int_{t_{a}}^{t_{b}} \mathbf{A}(s) d s\right) e^{-i \int_{t_{a}}^{t_{b}}\|\mathbf{A}(s)\|^{2} d s}
$$

More precisely, if:

- for all $t \in\left[0, t_{b}\right), \mathbf{r}_{\alpha}^{+}-\int_{t}^{t_{b}} \mathbf{A}(s) d s \notin \Gamma^{+}$, then

$$
u_{2}\left(\mathbf{r}_{\alpha}^{+}, t_{b}\right)=0
$$

- there exists $t_{i} \leqslant t_{a}$, such that $\mathbf{r}_{\alpha}^{+}-\int_{t_{i}}^{t_{b}} \mathbf{A}(s) d s \in \Gamma^{+}$, we denote

$$
\bar{t}:=\sup \left\{t_{i} \in\left[0, t_{a}\right] \text {, s.t. } \exists \mathbf{r}_{\beta_{i}}^{+} \in \Gamma^{+} \text {, with } \mathbf{r}_{\alpha}^{+}-\int_{t_{i}}^{t_{b}} \mathbf{A}(s) d s=\mathbf{r}_{\beta_{i}}^{+}\right\}
$$

The value at time $t_{b}$, and in $\mathbf{r}_{\alpha}^{+}$is given by:

$$
u_{2}\left(\mathbf{r}_{\alpha}^{+}, t_{b}\right)=w\left(\mathbf{r}_{\alpha}^{+}-\int_{\bar{t}}^{t_{b}} \mathbf{A}(s) d s, \bar{t}\right) e^{-i \int_{\bar{t}}^{t_{b}}\|\mathbf{A}(s)\|^{2} d s}
$$

- If there exists $t_{i} \in\left[t_{a}, t_{b}\right)$, such that there exists $\mathbf{r}_{\beta_{i}}^{+} \in \Gamma^{+}$such that

$$
\mathbf{r}_{\beta_{i}}^{+}=\mathbf{r}_{\alpha}^{+}-\int_{t_{i}}^{t_{b}} \mathbf{A}(s) d s
$$

Denoting:

$$
\tilde{t}:=\sup \left\{t_{i} \in\left[t_{a}, t_{b}\right), \text { s.t. } \exists \mathbf{r}_{\beta_{i}}^{+} \in \Gamma^{+}, \text {with } \mathbf{r}_{\alpha}^{+}-\int_{t_{i}}^{t_{b}} \mathbf{A}(s) d s=\mathbf{r}_{\beta_{i}}^{+}\right\}
$$

We denote by $\tilde{\mathbf{r}}_{\beta}^{+}$the corresponding $\mathbf{r}_{\beta}^{+}$. The value at time $t_{b}$, and in $\mathbf{r}_{\alpha}^{+}$is given by:

$$
u_{2}\left(\mathbf{r}_{\alpha}^{+}, t_{b}\right)=u_{2}\left(\tilde{\mathbf{r}}_{\beta}^{+}, \tilde{t}\right) e^{-i \int_{\tilde{t}}^{t_{b}}\|\mathbf{A}(s)\|^{2} d s}=w\left(\mathbf{r}_{\alpha}^{+}-\int_{\tilde{t}}^{t_{b}} \mathbf{A}(s) d s, \tilde{t}\right) e^{-i \int_{\tilde{t}}^{t_{b}}\|\mathbf{A}(s)\|^{2} d s}
$$

Repeat that in practice $w$ will be identified to $u_{1 \mid \Gamma^{-}}$. 
3. Finally at time $t_{b}$ the reconstruction solution is as follows, see Fig. 5:

$$
\left\{\begin{array}{l}
u_{1}\left(\mathbf{r}^{-}, t_{b}\right)=u_{2}\left(\mathbf{r}^{+}, t_{b}^{-}\right), \\
u_{2}\left(\mathbf{r}^{+}, t_{b}\right)=u_{1}\left(\mathbf{r}^{-}, t_{b}^{-}\right)
\end{array}\right.
$$

In length gauge the above approach could not be accurate as $[\triangle, \mathbf{r} \cdot \mathbf{E}] \neq 0$.

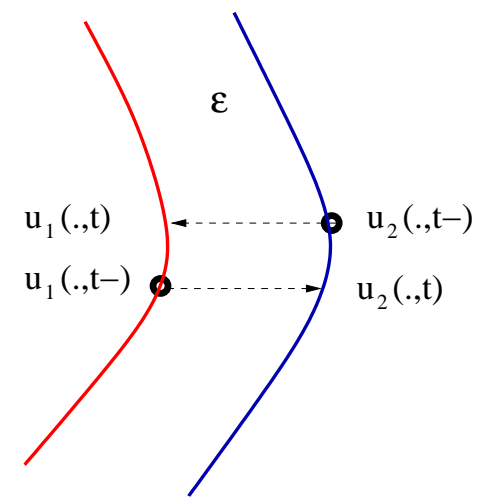

Figure 5: Final condition

\section{Numerical Approximation}

A Crank-Nicolson scheme in time is constructed to solve the TDSE [3]. Note that the key point in this paper is not the choice of the numerical solver but the construction of the transmission conditions. Naturally, the choice of the numerical method is guided by the type of PDE which is solved, including the boundary and transmission conditions. A real-space method, such as finite element, volume or difference methods is perfectly compatible with the chosen conditions, and we implement the most simple ones to illustrate the proposed method. Stability, convergence, and accuracy questions will not be detailed in this paper, but could be deduced from different references [6], [33], [3].

\subsection{Semi-discretized schemes}

We summarize here the general algorithm to update the solution from $t_{n}$ to $t_{n+1}$, with a time step denoted $\Delta t_{n}$. The details of the actual spatial discretization of the differential equation and boundary/transmission conditions will be presented in the following sections. Assume that $u_{1}$ and $u_{2}$ are known at time $t_{n}$ and Schwarz iteration $k-1$, and respectively denoted by $u^{n, k-1}$ and $v^{n, k-1}$. For instance, in length gauge:

1. In $\Omega_{1}$

$$
\left\{\begin{aligned}
u^{n+1, k}= & u^{n, k}+\frac{i \Delta t_{n}}{2} \Delta u^{n+1, k}+\frac{i \Delta t_{n}}{2} \Delta u^{n, k}-\frac{i \Delta t_{n}}{2} V_{c}\left(u^{n+1, k}+u^{n, k}\right) \\
& -i \frac{\Delta t_{n}}{2} \mathbf{r} \cdot \mathbf{E}^{n} u^{n, k}-i \frac{\Delta t_{n}}{2} \mathbf{r} \cdot \mathbf{E}^{n+1} u^{n+1, k} \text { in } \Omega_{1}, \\
\left(\partial_{\mathbf{n}^{-}}+B_{l, \Gamma^{-}}^{+, n+1}\right) u^{n+1, k}= & \left(\partial_{\mathbf{n}^{-}}+B_{l, \Gamma^{-}}^{+, n+1}\right) v^{n+1, k-1} \text { in } \Gamma^{-}
\end{aligned}\right.
$$


2. In $\Omega_{2}$

$$
\left\{\begin{aligned}
v^{n+1, k}= & v^{n, k}+\frac{i \Delta t_{n}}{2} \Delta v^{n+1, k}+\frac{i \Delta t_{n}}{2} \Delta v^{n, k}, \\
& -i \frac{\Delta t_{n}}{2} \mathbf{r} \cdot \mathbf{E}^{n} v^{n, k}-i \frac{\Delta t_{n}}{2} \mathbf{r} \cdot \mathbf{E}^{n+1} v^{n+1, k} \\
\left(\partial_{\mathbf{n}^{+}}+B_{l, \Gamma^{+}}^{+, n+1}\right) v^{n+1, k}= & \left(\partial_{\mathbf{n}^{+}}+B_{l, \Gamma^{+}}^{+, n+1}\right) u^{n+1, k-1} \text { on } \Gamma^{+}, \\
\left(\partial_{\mathbf{n}}+B_{l, \Sigma}^{+, n+1}\right) v^{n+1, k}= & 0 \text { on } \Sigma
\end{aligned}\right.
$$

Remark 3.1. We present the semi-discretized version of the method avoiding numerical computation in $\Omega_{2}$ and using the velocity gauge, by treating electrons in $\Omega_{2}$ as classical particles. Recall that in that case, no Schwarz algorithm is used and that $\Omega_{1} \cap \Omega_{2}=\emptyset$, see Section 2.3.

1. In $\Omega_{1}$

$$
\left\{\begin{aligned}
u^{n+1}= & u^{n}+\frac{i \Delta t_{n}}{2} \Delta u^{n+1}+\frac{i \Delta t_{n}}{2} \Delta u^{n}-\frac{i \Delta t_{n}}{2} V_{c}\left(u^{n+1}+u^{n}\right) \\
& -\Delta t_{n} \mathbf{A}^{n} \cdot \nabla u^{n}-i \frac{\Delta t_{n}}{2}\left(\mathbf{A}^{n}\right)^{2} u^{n}-i \frac{\Delta t_{n}}{2}\left(\mathbf{A}^{n+1}\right)^{2} u^{n+1} \text { in } \Omega_{1}, \\
\partial_{\mathbf{n}^{-}} u^{n+1^{-}}+B_{v, \Gamma^{-}}^{n+1^{-}} u^{n+1^{-}}= & 0 \text { in } \Gamma^{-}
\end{aligned}\right.
$$

2. For $\mathbf{r}^{+} \in \Gamma^{+}$, and $\mathbf{r}^{-}$corresponding point in $\Gamma^{-}$. If

- $\mathbf{A}^{n} \cdot \mathbf{n}^{+}<0$, then in $\mathbf{r}^{+}$:

$$
\left\{\begin{array}{l}
u^{n+1}\left(\mathbf{r}^{-}\right)=u^{n+1^{-}}\left(\mathbf{r}^{-}\right) \\
v^{n+1}\left(\mathbf{r}^{+}\right)=u^{n+1^{-}}\left(\mathbf{r}^{-}\right)
\end{array}\right.
$$

- $\mathbf{A}^{n} \cdot \mathbf{n}^{+}>0$, then in $\mathbf{r}^{+}$and $\mathbf{r}^{-}$:

$$
\left\{\begin{array}{l}
v^{n+1}\left(\mathbf{r}^{+}\right)=u^{n+1^{-}}\left(\mathbf{r}^{-} \int_{t_{n}}^{t_{n+1}} \mathbf{A}(s) d s\right) \\
u^{n+1}\left(\mathbf{r}^{-}\right)=v^{n+1^{-}}\left(\mathbf{r}^{+}\right)
\end{array}\right.
$$

where $v^{n+1^{-}}\left(\mathbf{r}^{-}-\int_{t_{n}}^{t_{n+1}} \mathbf{A}(s) d s\right)$ is computed according to the algorithm developed in Section 2.3.

\subsection{Cartesian coordinates in 1-d}

In the 1 -d case, we denote $\Omega_{1}=(a, b+\varepsilon)$ and $\Omega_{2}=(b, c)$ and by $h_{1,2}$ the space step in $\Omega_{1,2}$, and $\varepsilon$ is a (small) parameter, corresponding to the size of the overlapping region. In velocity gauge, the time step at time $t_{n}, \Delta t_{n}$ is determined according to the CFL condition $\Delta t_{n}\left|A\left(t_{n}\right)\right| \leqslant h_{1}$. No CFL condition is a priori necessary in length gauge, as the chosen discretization for boundary conditions is proven to be unconditionally stable [6]. In $\Omega_{2}$, we can choose a space step $h_{2} \gg h_{1}$. For consistency, the same time step as in $\Omega_{1}$ is used. In the following we denote $E^{n}=E\left(t_{n}\right)$. We denote by $\left(x_{j}\right)_{j \in\left\{1, \cdots, N_{1}\right\}}$ the grid nodes to $\Omega_{1}$ and $\left(y_{j}\right)_{j \in\left\{1, \cdots, N_{2}\right\}}$ those to $\Omega_{2}$. It it important to note that in the following, the domains overlap on 2 nodes such that: $x_{N_{1}}=y_{2}$ and $x_{N_{1}-1}=y_{1}$. For the sake of simplicity, in the rest of the presentation and in most of the numerical tests (except Test 2.b), we will assume that $h_{1}=h_{2}$ and then $\varepsilon=h_{1}$. The solution in $\Omega_{1}$ is denoted by $u$ and $v$ in $\Omega_{2}$. The numerical scheme which is chosen is a 3-point stencil Crank-Nicolson method which writes for interior points of $(a, b)$ as follows: for $1<j<N_{1}$ and $k \geqslant 1$,

$$
\begin{aligned}
\frac{u_{j}^{n+1, k}-u_{j}^{n, k}}{\Delta t_{n}}= & \frac{i}{2 h_{1}^{2}}\left(u_{j+1}^{n+1, k}-2 u_{j}^{n+1, k}+u_{j-1}^{n+1, k}\right)+\frac{i}{2 h_{1}^{2}}\left(u_{j+1}^{n, k}-2 u_{j}^{n, k}+u_{j-1}^{n, k}\right) \\
& -\frac{i}{2} V_{j}\left(u_{j}^{n+1, k}+u_{j}^{n, k}\right)-\frac{i}{2} x_{j} E^{n} u_{j}^{n, k}-\frac{i}{2} x_{j} E^{n+1} u_{j}^{n+1, k}
\end{aligned}
$$




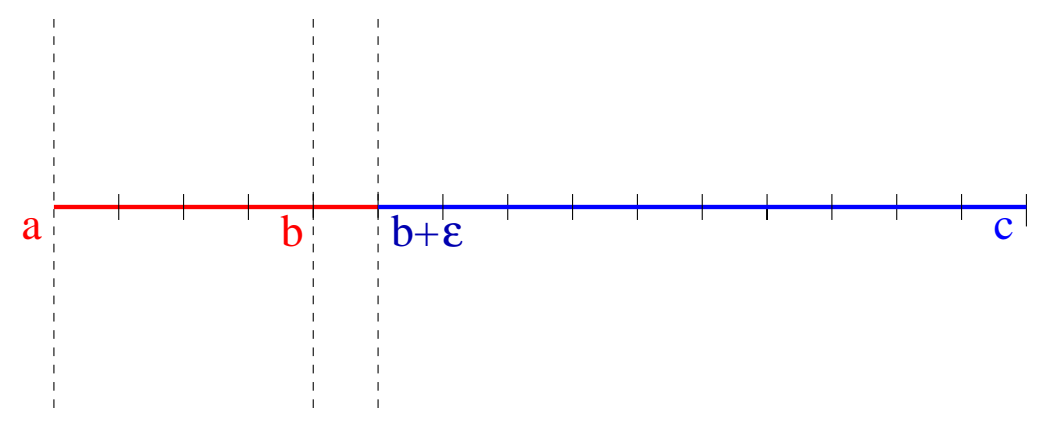

Figure 6: 1-d mesh

where $V_{j}=V_{c}\left(x_{j}\right)$, and in $\Omega_{2}$, for $1<j<N_{2}$ and $k \geqslant 1$ :

$$
\begin{aligned}
\frac{v_{j}^{n+1, k}-v_{j}^{n, k}}{\Delta t_{n}}= & \frac{i}{2 h_{2}^{2}}\left(v_{j+1}^{n+1, k}-2 v_{j}^{n+1, k}+v_{j-1}^{n+1, k}\right)+\frac{i}{2 h_{2}^{2}}\left(v_{j+1}^{n, k}-2 v_{j}^{n, k}+v_{j-1}^{n, k}\right) \\
& -\frac{i}{2} y_{j} E^{n} v_{j}^{n, k}-\frac{i}{2} y_{j} E^{n+1} v_{j}^{n+1, k}
\end{aligned}
$$

Discretization of boundary conditions for velocity gauge. As described above at $\Gamma^{+}\left(\operatorname{resp} . \Gamma^{-}\right)$ boundary conditions are simultaneously outgoing and incoming non-reflecting boundary conditions:

- On $\Gamma^{-}$. At $x=x_{N_{1}}=y_{2}=b$, the chosen non-reflecting boundary condition [8] writes

$$
\left(\partial_{x}+B_{v, \Gamma^{-}}^{+, n+1}\right) u^{n+1, k}\left(x_{N_{1}}\right)=\left(\partial_{x}+B_{v, \Gamma^{-}}^{-, n+1}\right) v^{n+1, k-1}\left(y_{2}\right),
$$

where $B_{v, \Gamma^{-}}^{+}:=i \Lambda_{1 / 2}^{+}+i \Lambda_{0}^{+}+i \Lambda_{-1 / 2}^{+}+\ldots$ We truncate the series at $\Lambda_{-1 / 2}^{+}$considering large time frequency regime. In terms of symbols, denoting $a=\sigma(A)$ and $v_{c}=\sigma\left(V_{c}\right)$, it can be proved [8] that:

$$
\lambda_{1 / 2}^{+}=-\sqrt{-\tau}, \lambda_{0}^{+}=\frac{1}{2 \lambda_{1 / 2}^{+}}\left(-i \partial_{x} \lambda_{1 / 2}^{+}+a \lambda_{1 / 2}^{+}\right),
$$

and

$$
\lambda_{-1 / 2}^{+}=\frac{1}{2 \lambda_{1 / 2}^{+}}\left(-v_{c}-a^{2}-i \partial_{x} \lambda_{0}^{+}+a \lambda_{0}^{+}-\left(\lambda_{0}^{+}\right)^{2}+i \partial_{\tau} \lambda_{1 / 2}^{+} \partial_{t} \lambda_{1 / 2}^{+}\right)
$$

This leads in terms of operators to

$$
\left\{\begin{aligned}
\Lambda_{1 / 2}^{+} u\left(x_{N_{1}}, t_{n+1}\right) & =-e^{i \pi / 4} \partial_{t} \int_{0}^{t} \frac{u\left(x_{N_{1}}, s\right)}{\sqrt{\pi\left(t_{n+1}-s\right)}} d s\left(t=t_{n+1}\right) \\
\Lambda_{0}^{+} u\left(x_{N_{1}}, t_{n+1}\right) & =\frac{1}{2} A\left(t_{n+1}\right) u\left(x_{N_{1}}, t_{n+1}\right) \\
\Lambda_{-1 / 2}^{+} u\left(x_{N_{1}}, t_{n+1}\right) & =e^{i \pi / 4} \frac{4 V_{c}\left(x_{N_{1}}\right)+3 A^{2}\left(t_{n+1}\right)}{8} \int_{0}^{t_{n+1}} \frac{u\left(x_{N_{1}}, s\right)}{\sqrt{\pi\left(t_{n+1}-s\right)}} d s
\end{aligned}\right.
$$

At Schwarz iteration $k$, this is discretized by

$$
u_{N_{1}}^{n+1, k}=u_{N_{1}-1}^{n+1, k}-h_{1} B_{v, \Gamma^{-}}^{+, n+1} u^{n+1, k}\left(x_{N_{1}}\right)+v_{2}^{n+1, k-1}-v_{1}^{n+1, k-1}+h_{2} B_{v, \Gamma^{+}}^{-, n+1} v^{n+1, k-1}\left(y_{2}\right)
$$

More precisely, using [6], and say in the field-free case, we the DtN operator is discretized as

$$
\left\{\begin{aligned}
u_{N_{1}}^{n+1, k}\left(1+\beta^{n+1}\right)-\beta^{n+1} u_{N_{1}-1}^{n+1, k}= & -\beta^{n+1} \sum_{l=1}^{n+1} \alpha_{l} \frac{u_{N_{1}}^{n+1-l}-u_{N_{1}-1}^{n+1-l}}{h_{1}} \\
& +v_{2}^{n+1, k-1}\left(1+\beta^{n+1}\right)-\beta^{n+1} v_{1}^{n+1, k-1} \\
& +\beta^{n+1} \sum_{l=1}^{n+1} \alpha_{l} \frac{v_{2}^{n+1-l}-v_{1}^{n+1-l}}{h_{2}}
\end{aligned}\right.
$$


where $\beta^{n+1}=e^{i \pi / 4} \sqrt{\Delta t_{n+1} / 2}$ and $\left(\alpha_{l}\right)_{l}$ is a sequence of real numbers defined in [6]. Coupled with a Crank-Nicholson scheme, this proposed discretization allows for an unconditionally stable in the potential-free case. Note that in (9), the terms $\sum_{l=1}^{n+1} \alpha_{l} \partial_{x} v^{n+1-l}\left(y_{2}\right)$ and $\sum_{l=1}^{n+1} \alpha_{l} \partial_{x} u^{n+1-l}\left(x_{N_{1}}\right)$ are evaluated from times $t_{n}$ and earlier ones.

- On $\Gamma^{+}$. By symmetry we can deduce the boundary condition on $\Gamma^{+}$. At $y=y_{1}=x_{N_{1}-1}$, that is for $j=1$ the boundary condition writes

$$
\left(\partial_{x}+B_{v, \Gamma^{+}}^{+, n+1}\right) v^{n+1, k}\left(y_{1}\right)=\left(\partial_{x}+B_{v, \Gamma^{+}}^{+, n+1}\right) u^{n+1, k-1}\left(x_{N_{1}-1}\right)
$$

with $B_{v, \Gamma^{+}}^{-, n+1}:=i \Phi_{1 / 2}^{+}+i \Phi_{0}^{+}+i \Phi_{-1 / 2}^{+}+\ldots$ with $\Phi_{1 / 2-i}^{+}=\Lambda_{1 / 2-i}^{+}, i \in \mathbb{N} / 2$. This is discretized at Schwarz iteration $k$, by

$$
v_{1}^{n+1, k}=v_{2}^{n+1, k}-h_{2} B_{v, \Gamma^{+}}^{-} v^{n+1, k}\left(y_{1}\right)+u_{N_{1}-1}^{n+1, k-1}-u_{N_{1}}^{n+1, k-1}+h_{2} B_{v, \Gamma^{-}}^{+} u^{n+1, k-1}\left(x_{N_{1}-1}\right)
$$

- On $\Sigma$. Finally at $y=y_{N_{2}}=c$, that is for $j=N_{2}$

$$
\left(\partial_{x}+B_{v, \Sigma}\right) v^{n+1}=0
$$

where $B_{v, \Sigma}^{+}:=i \Lambda_{1 / 2}+i \Lambda_{0}+i \Lambda_{-1 / 2}+\ldots$ with

$$
\left\{\begin{aligned}
\Lambda_{1 / 2} v\left(c, t_{n+1}\right) & =-e^{i \pi / 4} \partial_{t} \int_{0}^{t} \frac{v(c, s)}{\sqrt{\pi\left(t_{n+1}-s\right)}} d s\left(t=t_{n+1}\right), \\
\Lambda_{0} v\left(c, t_{n+1}\right) & =\frac{1}{2} A\left(t_{n+1}\right) v\left(c, t_{n+1}\right) \\
\Lambda_{-1 / 2} v\left(c, t_{n+1}\right) & =e^{i \pi / 4} \frac{3 A^{2}\left(t_{n+1}\right)}{4} \int_{0}^{t_{n+1}} \frac{v(c, s)}{\sqrt{\pi\left(t_{n+1}-s\right)}} d s
\end{aligned}\right.
$$

The convergence of the Schwarz method is naturally dependent on the order of approximation of $\lambda^{+}$. More specifically, the convergence factor is expected to be of the form $\left|e^{-2 i \int_{0}^{\varepsilon} \lambda^{+}\left(x^{\prime}, t\right) d x^{\prime}}\right|$, where $\varepsilon$ is the size of the overlapping region. The approximation of $\lambda^{+}$, by say $\tilde{\lambda}^{+}$, should then be designed such that $\left|e^{-2 i \int_{0}^{\varepsilon} \tilde{\lambda}^{+}\left(x^{\prime}, t\right) d x^{\prime}}\right|$ is as small as possible, and at the same time such that the approximation of the transmission condition, involving $\partial_{x}+i \tilde{\Lambda}^{+}$, is as simple (as well as stable) as possible.

Discretization of boundary conditions for length gauge. In that situation, we can easily check that the corresponding pseudodifferential operators are of the form [6]

$$
\lambda_{1 / 2}^{+}=-\sqrt{-\tau}, \quad \lambda_{0}^{+}=-\int_{0}^{t} E(s) d s-t V_{c}^{\prime}(x), \quad \lambda_{-1 / 2}^{+}=0, \quad \lambda_{-1}^{+}=-i \frac{E(t)+V_{c}^{\prime}(x)}{4 \tau}
$$

with in particular on $\Gamma^{ \pm}$or $\Sigma$

$$
\Lambda_{0}^{+} u(x, t)=-\left(\int_{0}^{t} E(s) d s+t V_{c}^{\prime}(x)\right) u(x, t), \quad \Lambda_{-1}^{+} u(x, t)=-\frac{E(t)+V_{c}^{\prime}(x)}{4} \int_{0}^{t} u(x, s) d s
$$

In that gauge, and as proposed in [7], an unitary transformation can be used to generate a new condition of the form: $\partial_{x} u+e^{-i \pi / 4} e^{i \int_{0}^{t} V_{c}(x)+x E(s) d s} \partial_{t}^{1 / 2}\left(e^{-i \int_{0}^{t} V_{c}(x)+x E(s) d s} u\right)=0$, which includes the contribution of $\lambda_{0}^{+}$, and which is easy to discretized from (9).

Remark 3.2 (Localized transmission boundary conditions). Let us remark here that more local transmission boundary conditions could be used in practice. For example, we refer to [7] where rational approximants are used to approximate the nonlocal fractional operators. This implies a gain in terms of computational cost. The price to pay is that it is not clear if the resulting scheme is stable or not (probably not) [7]. Similar results can be developed for the 2d case [9]. 


\subsection{Polar coordinates in 2-d}

We here give some basic information about the implementation of the presented method in higher dimension, more specifically the case of 2 -d polar coordinates. The computation domain $\Omega$ is decomposed in two regions $\Omega_{1}=C\left(\mathbf{0}, R_{1}+\varepsilon\right)$ a disc centered in $\mathbf{0} \in \mathbb{R}^{2}$ of radius $R_{1}+\varepsilon, \varepsilon>0, \Omega_{2}=D\left(\mathbf{0}, R_{1}\right.$, $\left.R_{2}\right)$, where $D$ is a crown of radii $R_{1}, R_{2}$, with $R_{2}>R_{1}$. Boundary $\Sigma$ is the circle centered in 0 and radius $R_{2}$. The chosen gauge here is the velocity one. In cylindrical coordinates, the TDSE that we consider writes:

$$
\begin{aligned}
i \partial_{t} u(r, \theta, t)= & -\left(\partial_{r}^{2}+\frac{1}{r} \partial_{r}+\frac{1}{r^{2}} \partial_{\theta}^{2}\right) u(r, \theta, t)+V_{c}(r) u(r, \theta, t)+V(r) u(r, \theta, t) \\
& -i A_{r}(t) \partial_{r} u(r, \theta, t)-\frac{i}{r} A_{\theta}(t) \partial_{\theta} u(r, \theta, t)+\|\mathbf{A}\|^{2} u(r, \theta, t)+\frac{A_{r}(t)}{r} u(r, \theta, t)
\end{aligned}
$$

where $\|\mathbf{A}\|^{2}=A_{\theta}^{2}+A_{r}^{2}$.

For the sake of simplicity, the numerical approach which is chosen is a finite difference scheme, although more advanced techniques are still applicable within the framework of this paper. In $\Omega_{1}$, a fine mesh, $\tau\left(\Omega_{1}\right)$ is used to spatially discretized the equation. In $\Omega_{k}(k=1,2)$, the discretization points in $\theta$ are denoted $\left(\theta_{j}\right)_{j=1, \cdots, N_{\theta}}$,

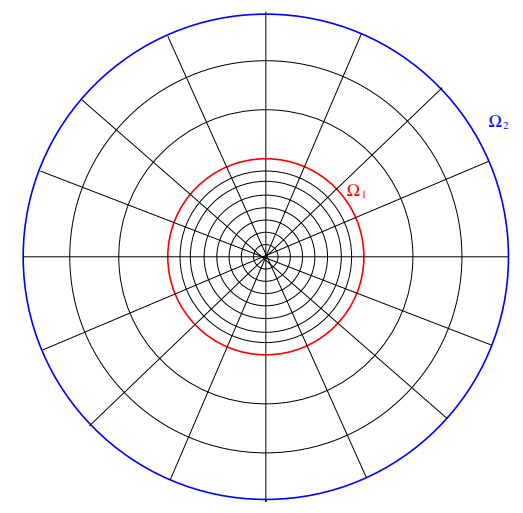

Figure 7: Polar coordinates

and by $\left(r_{j}^{k}\right)_{j=1, \cdots, N_{r}^{k}}$ in $r$. Space steps are denoted by $\Delta r_{k}, \Delta \theta$ for $k=1,2$. Finite differences are used to approximate the equation. The solution $u$ (resp. $v$ ) at $\left(r_{i}^{k}, \theta_{j}\right)$, with $i=1, \cdots, N_{r}^{k}, j=1, \cdots, N_{\theta}^{(1)}$, is approximated by $u_{i, j}$. Electric potential values are given by $A_{r}\left(t_{n}\right), A_{\theta}\left(t_{n}\right)$. We first describe the interior scheme. As before an upwinding is necessary to get a stable discretization of transport terms: $A_{r}(t) \partial_{r} u$ and $A_{\theta}(t) \partial_{\theta}:$

$$
\left\{\begin{array}{lll}
A_{r}\left(t_{n}\right) \partial_{r} u_{i, j}^{n, k} & \sim \frac{u_{i, j}^{n}-u_{i-1, j}^{n, k}}{\Delta r_{i}} & \text { if } A_{\theta}\left(t_{n}\right)>0, \\
A_{r}\left(t_{n}\right) \partial_{r} u_{i, j}^{n, k} & \sim \frac{u_{i+1, j}^{n}-u_{i, j}^{n, k}}{\Delta r_{i}} & \text { if } A_{\theta}\left(t_{n}\right)<0
\end{array}\right.
$$

and

$$
\begin{cases}\frac{A_{\theta}\left(t_{n}\right)}{r} \partial_{\theta} u_{i, j}^{n, k} \sim \frac{u_{i, j}^{n}-u_{i, j-1}^{n, k}}{r_{i} \Delta \theta}, & \text { if } A_{\theta}\left(t_{n}\right)>0, \\ \frac{A_{\theta}\left(t_{n}\right)}{r} \partial_{\theta} u_{i, j}^{n, k} \sim \frac{u_{i, j+1}^{n}-u_{i, j}^{n, k}}{r_{i} \Delta \theta}, & \text { if } A_{\theta}\left(t_{n}\right)<0\end{cases}
$$

For the other terms any classical approach can be used. The operators $i / r \partial_{r}$ and $1 / r \partial_{\theta}$ are usually approximated using a centered scheme and the other terms are approximated using a classical semi-implicit 
Crank-Nicolson approach as in the 1-d case. Special treatment is of course necessary at $r=0$.

We now specify the boundary conditions $\mathcal{B}_{v, \Gamma^{-}}, \mathcal{B}_{v, \Gamma^{+}}, \mathcal{B}_{v, \Sigma}$ in polar coordinates. Then we will discuss their discretization on $\Gamma^{-}=C\left(\mathbf{0}, R_{1}+\varepsilon\right), \Gamma^{+}=C\left(\mathbf{0}, R_{1}\right), \Sigma=C\left(\mathbf{0}, R_{2}\right)$.

- On $\Gamma^{-}$. According to [8], the boundary conditions is given in that case by

$$
\left(\partial_{\mathbf{n}^{-}}+B_{\Gamma^{-}}^{+}\right) u^{k}=\left(\partial_{\mathbf{n}^{-}}+B_{\Gamma^{-}}^{+}\right) v^{k-1}
$$

where $B_{\Gamma^{-}}^{+}:=i \Lambda_{1 / 2}^{+}+i \Lambda_{0}^{+}+i \Lambda_{-1 / 2}^{+}+\ldots$ which have symbols described in [8]. In the later, several strategies are proposed to construct and approximate these pseudodifferential symbols, which are given for high frequency regimes, by

$$
\left\{\begin{array}{lll}
\Lambda_{1 / 2}^{+} & \sim-e^{i \pi / 4} \partial_{t}^{1 / 2}+e^{i \pi / 4} \frac{1}{2} \partial_{\theta}^{2} \partial_{t}^{-1 / 2} \\
\Lambda_{0}^{+} & \sim-\frac{i}{2\left(R_{1}+\varepsilon\right)}+\frac{A_{r}}{2}+\frac{i e^{i \pi / 4} A_{\theta}}{2\left(R_{1}+\varepsilon\right)} \partial_{\theta} \partial_{t}^{-1 / 2}+\frac{i}{2\left(R_{1}+\varepsilon\right)} \partial_{\theta}^{2} \partial_{t}^{-1} \\
\Lambda_{-1 / 2}^{+} & \sim \frac{e^{i \pi / 4}}{8\left(R_{1}+\varepsilon\right)^{2}} \partial_{t}^{-1 / 2}-e^{i \pi / 4}\left(\frac{V_{c}+\|\mathbf{A}\|^{2}}{2}+\frac{A_{r}}{R_{1}+\varepsilon}\right) \partial_{t}^{-1 / 2}
\end{array}\right.
$$

The use of these ABC for domain decomposition will be studied in a future paper.

- On $\Gamma^{+}$. Similarly, the boundary condition is given in that case by

$$
\left(\partial_{\mathbf{n}^{+}}+B_{\Gamma^{+}}^{+}\right) v^{k}=\left(\partial_{\mathbf{n}^{+}}+B_{\Gamma^{+}}^{+}\right) u^{k-1}
$$

which is evaluated at $r=R_{1}$.

- On $\Sigma$, the boundary condition is similar to the one in $\Gamma^{-}$, except that $V_{c}$ is set to zero, and writes:

$$
\left(\partial_{\mathbf{n}_{\Sigma}}+B_{\Sigma}^{+}\right) v^{k}=0
$$

with $B_{\Sigma}^{+}$similar to $B_{\Gamma^{-}}^{+}$except that $V_{c}$ is set to 0 and $R_{1}+\varepsilon$ is replaced by $R_{2}$.

\section{Numerical Results}

This section is devoted to some numerical simulations illustrating the proposed method in 1-d. We compare the solution of reference $u_{r}$ for the TDSE i) in Domain $\Omega$, where absorbing boundary conditions are imposed on $\Sigma$ with ii) the reconstructed solution $u_{h}$, using the derived method in $\Omega_{1}, \Omega_{2}$ with transmission/absorbing boundary conditions on $\Gamma^{ \pm}$and $\Sigma$. For completeness, a 3-subdomain test is also proposed (Test 3). First, we shortly discuss the computational complexity as well the data storage related to the method. When the total number of discretization points $N$ (excluding the boundary conditions) in $\Omega$ is equal to the sum $N_{1}+N_{2}$ of discretization points $N_{1}$ in $\Omega_{1}$ and $N_{2}$ in $\Omega_{2}$, we can easily evaluate the computational efficiency. Indeed, the computational cost of one time step of the interior scheme is roughly in $\mathcal{O}\left(N^{\alpha}\right)$ for $u_{r}$ (due to the need for solving a linear system coming from the Crank-Nicolson discretization in time) for some $\alpha>1$. By comparison, the computation of interior points for $u_{h}$ necessitates $\mathcal{O}\left(N_{1}^{\alpha}+N_{2}^{\alpha}\right)$ operations. When $N \gg N_{1}+N_{2}$, we then expect a large improvement in term of computational complexity as $N_{1}^{\alpha}+N_{2}^{\alpha} \ll N^{\alpha}$. Naturally, this method only makes sense for parallel computing.

In order to be relevant, from a computational complexity view point, the Schwarz algorithm should converge in few iterations. This is precisely why high-order absorbing boundary conditions are used.

Test 1 This first test is devoted to the evolution of 2 wavepackets (see Fig. 8) propagating in opposite directions from time 0 to $T_{f}=7$. The parameters for this first computation are the following

- $a=-80, c=80, b=20$ with $N=800, N_{1}=500, N_{2}=300$. 


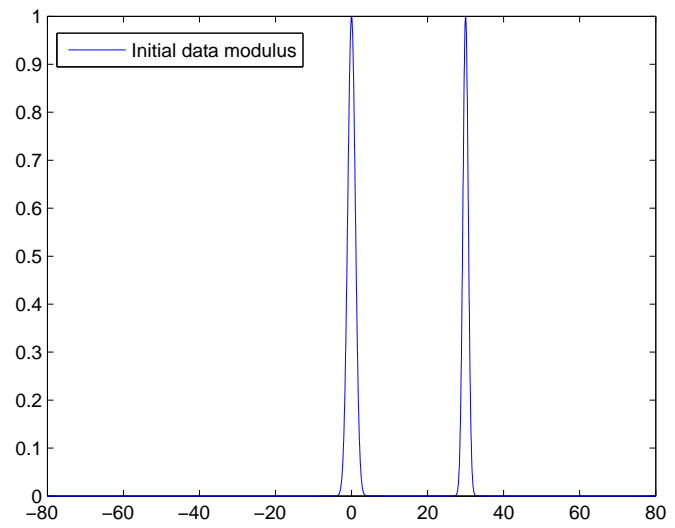

Figure 8: Modulus of initial data

- The time step is fixed to $\Delta t=0.05$.

- We impose the incoming/outgoing boundary conditions described above, with $E(t)=V_{c}(x)=0$, at $b$, and compare the modulus of the reconstructed solution $(v$ in $(a, b)$ and $w$ in $(b, c))(v, w)$ with the solution of reference, computed in $(a, c)$.

- The overlapping region is reduced to two nodes.

- In the following the electric field is set to zero and the initial data is given by

$$
u_{0}(x)=\exp \left(-\frac{1}{2}\left(\frac{c+d}{2}-x\right)^{2}\right) \exp \left(i k_{0} x\right)+\exp \left(-\left(\frac{3 c}{8}-x\right)^{2}\right) \exp \left(-i k_{0} x\right)
$$

with $k_{0}=4$.

We do not expect any significant improvement in term of computational time as $N_{1}+N_{2}=N$ with $h_{1}=h_{2}$, but ensures that the method is accurate enough for 10 Schwarz iterations (see Fig. 9). This test is similar to the one proposed in [24].

Test 2.a The second test consists of the interaction of an electric field with a two-center molecule. In that case, in length gauge, the equation writes on $(-a, a)$ :

$$
i \partial_{t} u(x, t)=-\triangle u(x, t)+V_{c}(x) u+x E(t) u(x, t)
$$

with

$$
V_{c}(x)=-\frac{g}{\sqrt{(x-R / 2)^{2}+\varepsilon}}-\frac{g}{\sqrt{(x+R / 2)^{2}+\varepsilon}}
$$

and $R=2$ is the inter-nucleus distance and $\varepsilon$ is a regularizing potential parameter taken equal here to 0.2 . The initial data is the ground state of the two-center molecule, which is the eigenfunction associated to the smallest eigenvalue to $-\triangle+V_{c}$. We choose $g=0.5$. The electric potential is given by

$$
E(t)=E_{0} \exp \left(-\eta\left(t-\frac{T_{f}}{2}\right)^{2}\right) \sin \left(\frac{6 \pi}{T_{f}} t\right), \quad T_{f}=32, \quad E_{0}=0.1, \quad \eta=10^{-2}
$$

The field is represented in Fig 10. The numerical data for this first test are as follows: $\Omega=(-a, a)$, $\Omega_{1}=(-a, c+\varepsilon)$ and $\Omega_{2}=(c, a)$, with 

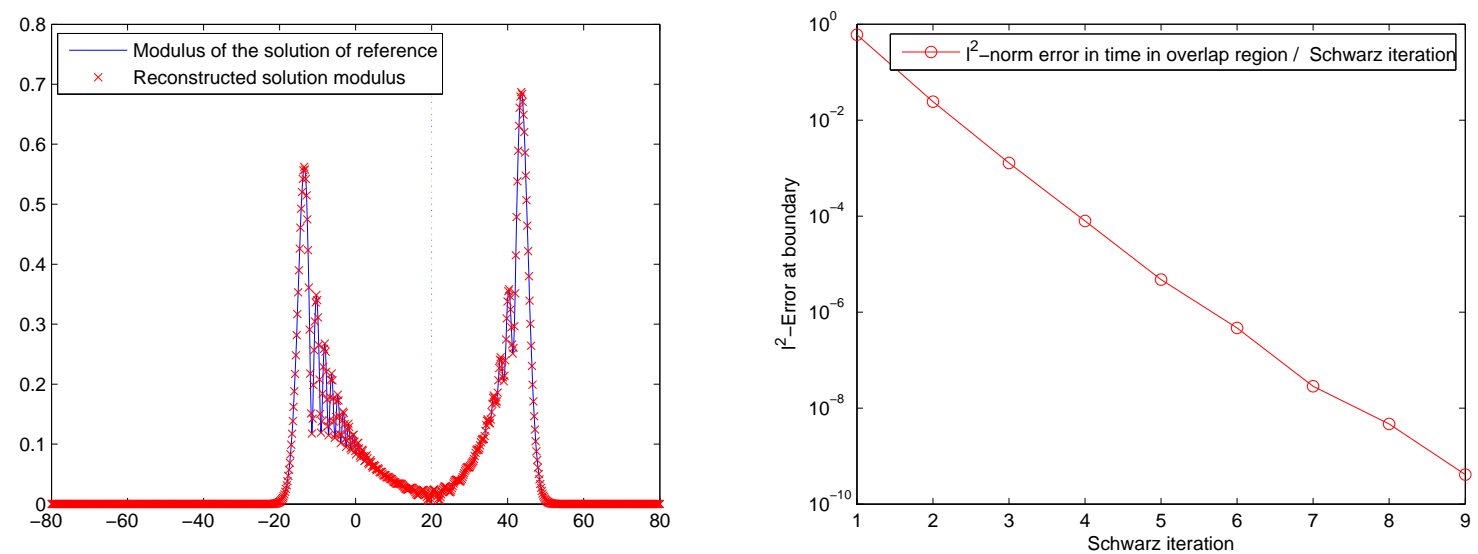

Figure 9: For $N=800$ and 9 Schwarz iterations, modulus of the reference and reconstructed solutions at i) $t=7$ and ii) $\ell^{2}$-norm error in time at boundary. The interface between the 2 subdomains is located at $x=20$

- $a=40, c=a / 4$.

- $N=400$ nodes to mesh $(-a, a)=\Omega, M=250$ nodes to mesh $\Omega_{1}=(-a, c+\varepsilon)$, and $M=150$ nodes to mesh $(c, a)=\Omega_{2}$. The step size in $\Omega, \Omega_{1}$ and $\Omega_{2}$ is then identical.

- $\left(x_{i}\right)_{i=\{1, \cdots, N\}},\left(y_{i}\right)_{i=\{1, \cdots, M\}}$ denote the mesh nodes respectively in $\Omega_{1}, \Omega_{2}$. The corresponding solutions are denoted by $v$ and $w$. The overlap region is reduced to 2 nodes: $x_{N}=y_{2}, x_{N-1}=y_{1}$.

- The time step is fixed to $\Delta t=0.05$.

We represent a solution of reference, computed in all $\Omega$, with the reconstructed solution $(v, w)$ from $\Omega_{1} \cup \Omega_{2}$. In addition, we represent the convergence graphs for the Schwarz algorithm. More specifically, we report

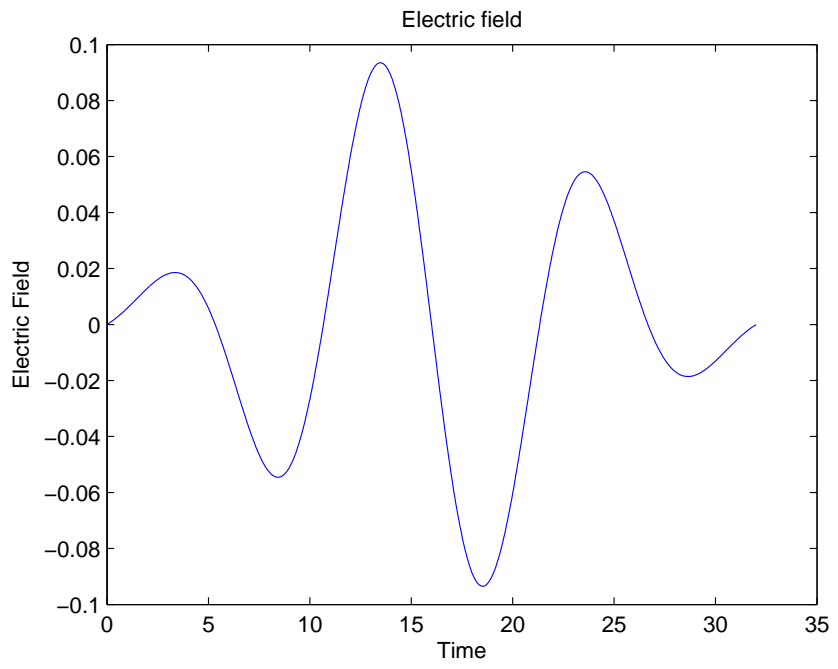

Figure 10: Electric field as a function of time.

the error between the reconstructed solution $u_{h}$ and the solution of reference (computed in all $(-a, a)$ ): $\left\|u_{h}-u_{r}\right\|_{\ell^{2}(-a, a)}$ as a function of the number of Schwarz iterations, at final time $T_{f}=32$, Fig. 13 (left). In 
Fig. 13 (right), we represent the $\ell^{2}$-norm in time of $v_{N}-w_{2}$, that is $\left(\Delta t \sum_{l=0}^{n}\left|v_{N}^{l}-w_{2}^{l}\right|^{2}\right)^{1 / 2}$.
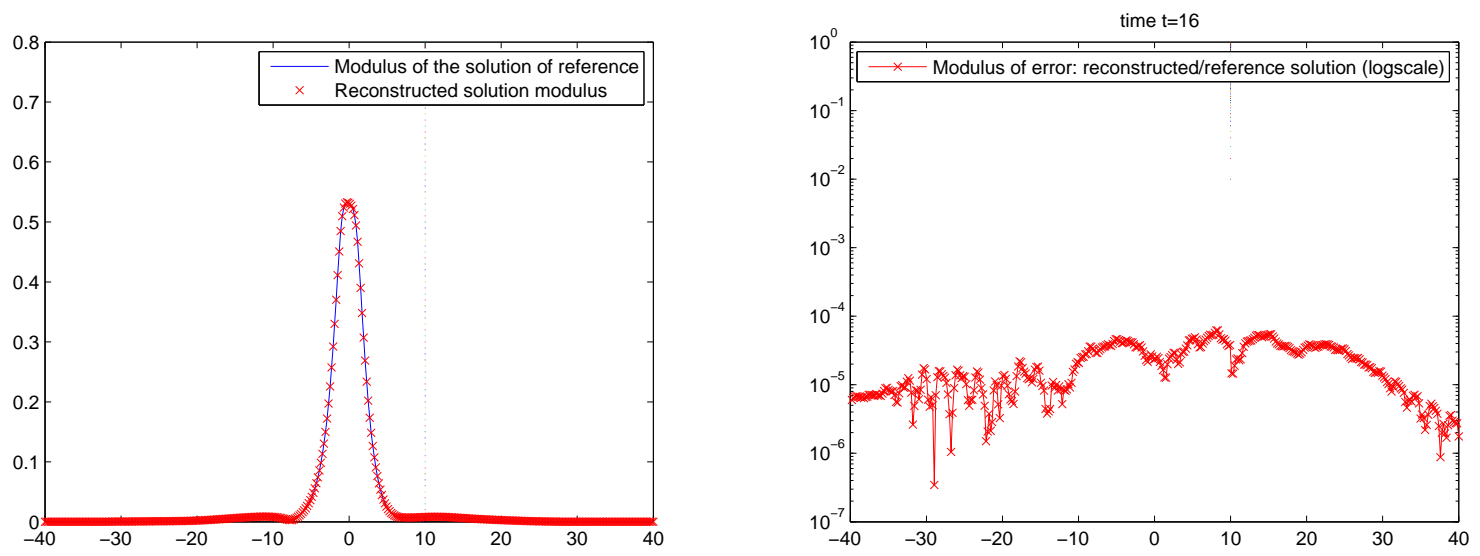

Figure 11: i) Comparison of the modulus of reconstruction solution with the modulus of the solution of reference (computed in all $\Omega$ ) and ii) error in logscale, at time 16 . The interface between the 2 subdomains is located at $x=10$
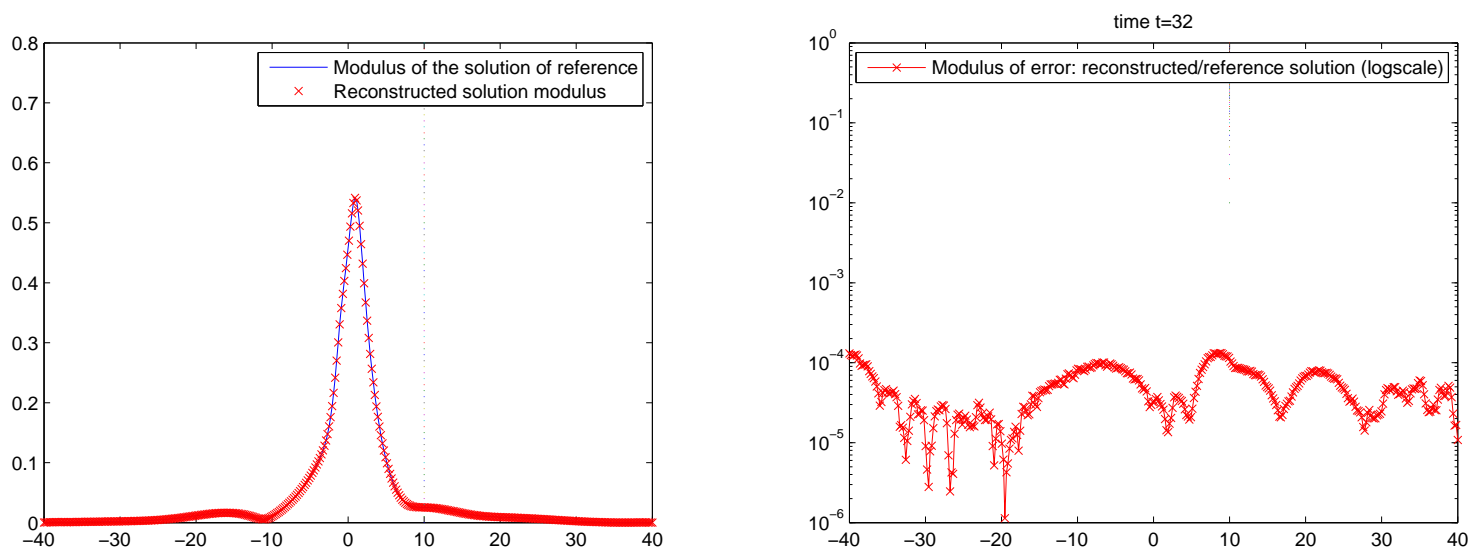

Figure 12: i) Comparison of the modulus of reconstruction solution with the modulus of the solution of reference (computed in all $\Omega$ ) and ii) errors in logscale, at time 32 . The interface between the 2 subdomains is located at $x=10$

Test 2.b This test is similar to the previous one, except that we now assume that $h_{2} \approx 5 h_{1}$. The computation in $\Omega_{2}$ is then accelerated by a factor $\approx 5^{\alpha}(\alpha \approx 3 / 2)$ compared to the computation in $\Omega_{2}$, in Test 2.a. We still compare the result with a solution of reference. All the other physical and numerical data are the same as in Test 2.a. We again compare the reconstructed solution $(v, w)$ to a solution of reference $u_{r}$. We represent the solution at time $t=T_{f}=32$ (see Fig. 14).

Test 2.c This test is similar to Test 2.a, except that the electric field is more intense and is defined by

$$
E(t)=E_{0} \exp \left(-\eta\left(t-\frac{T_{f}}{2}\right)^{2}\right) \sin \left(\frac{5 \pi}{T_{f}} t\right), \quad T_{f}=32, \quad E_{0}=0.6, \quad \eta=10^{-2}
$$

In addition, the numerical data are given by 

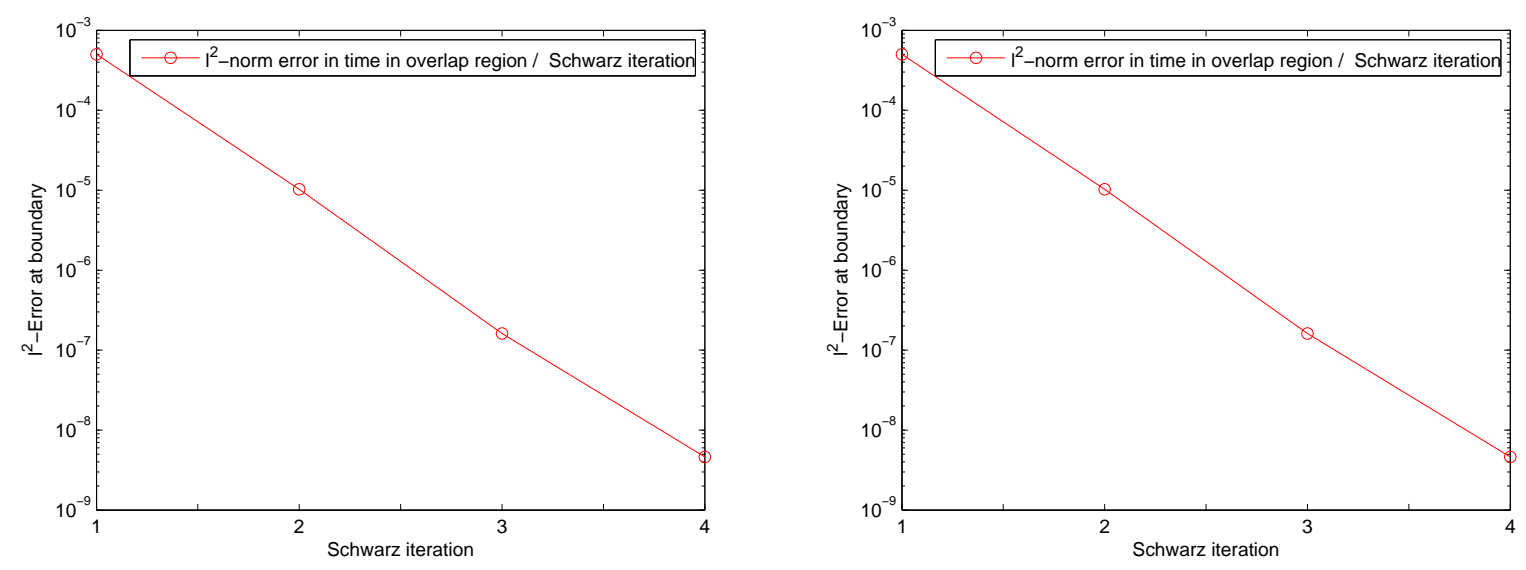

Figure 13: i) $\ell^{2}$-error in time at the boundary $\left\|v\left(x_{N}, \cdot\right)-w\left(y_{2}, \cdot\right)\right\|_{l^{2}\left(0, T_{f}\right)}$ as a function of Schwarz iterations at time $t=16$, ii) at time $t=32$

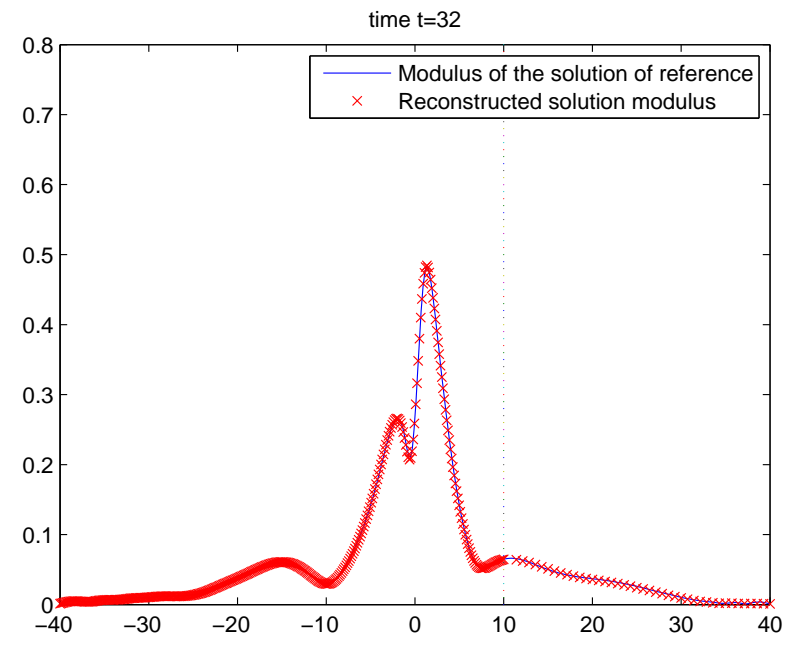

Figure 14: Comparison of the modulus of reconstruction solution $(v, w)$ with the modulus of the solution of reference $u_{r}$ (computed in all $\Omega$ ) at time $t=32$. Domain interface is located at $x=10$ and $h_{2} \approx 5 h_{1}$

- $a=60, c=a / 4$.

- $N=800$ nodes to mesh $(-a, a)=\Omega, 500$ nodes to mesh $\Omega_{1}=(-a, c+\varepsilon)$, and $M=300$ nodes to mesh $(c, a)=\Omega_{2}$. The step size in $\Omega, \Omega_{1}$ and $\Omega_{2}$ is then identical.

- $\left(x_{i}\right)_{i=\{1, \cdots, N\}},\left(y_{i}\right)_{i=\{1, \cdots, M\}}$ denote the mesh nodes respectively in $\Omega_{1}, \Omega_{2}$. The corresponding solutions are denoted $v, w$. The overlap region is reduced to 2 nodes: $x_{N}=y_{2}, x_{N-1}=y_{1}$.

We represent a solution of reference $u_{r}$, computed in all $\Omega$, with the reconstructed solution $(v, w)$ from $\Omega_{1} \cup \Omega_{2}$, at time $t=32$, as well as the $\ell^{2}$-norm in time of $v_{N}-w_{2}$, that is $\left(\Delta t \sum_{l=0}^{n}\left|v_{N}^{l}-w_{2}^{l}\right|^{2}\right)^{1 / 2}$ (see Figs. $15,16)$.

Test 2.d In this test, we compare the transmission condition $\left(\partial_{\mathbf{n}}+B^{(p)}\right) u_{1}^{(k)}=\left(\partial_{\mathbf{n}}+B^{(p)}\right) u_{2}^{(k-1)}$ at different order $(p=1,2,3)$, that is with i) $B^{(1)}:=i \Lambda_{1 / 2}^{+}$, ii) $B^{(2)}:=i\left(\Lambda_{1 / 2}^{+}+\Lambda_{0}^{+}\right)$, iii) $B^{(3)}:=i\left(\Lambda_{1 / 2}^{+}+\Lambda_{0}^{+}+\Lambda_{-1}^{+}\right)$ 

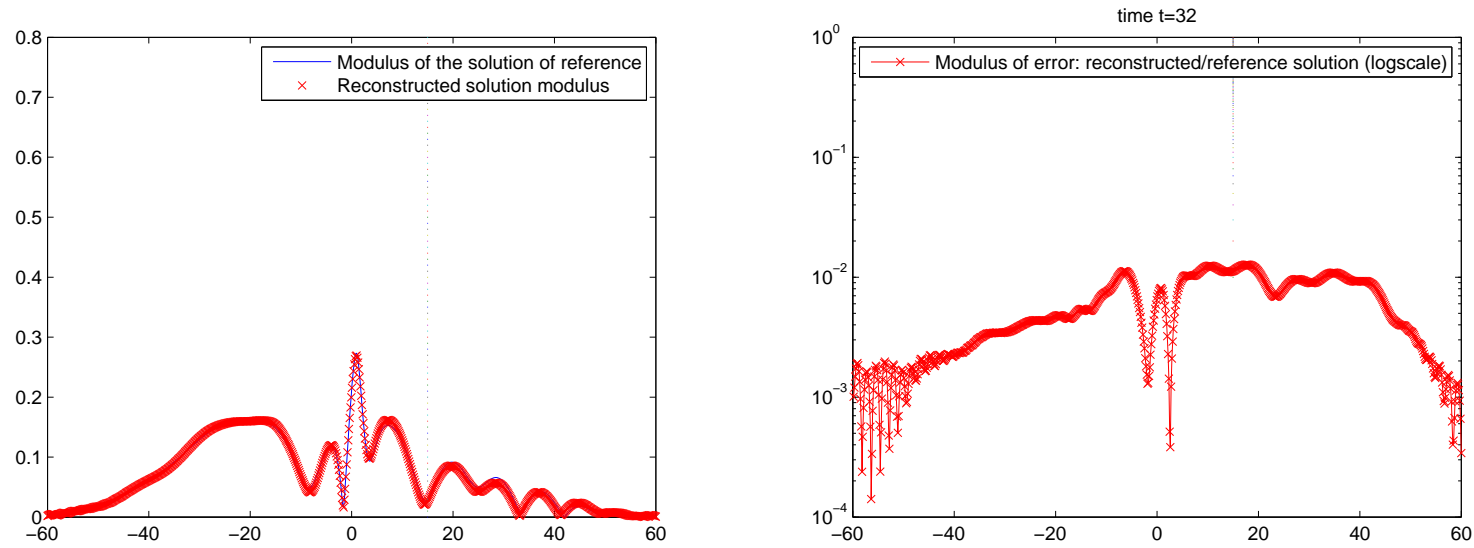

Figure 15: i) Comparison of the modulus of reconstruction solution with the modulus of the solution of reference (computed in all $\Omega$ ) at time $t=32$. Domain interface is located at $x=20$, ii) pointwise error between reconstructed solution and solution of reference. $\ell^{2}$-error in time at the boundary $\left\|v\left(x_{N}, \cdot\right)-w\left(y_{2}, \cdot\right)\right\|_{l^{2}\left(0, T_{f}\right)}$ as a function of Schwarz iterations

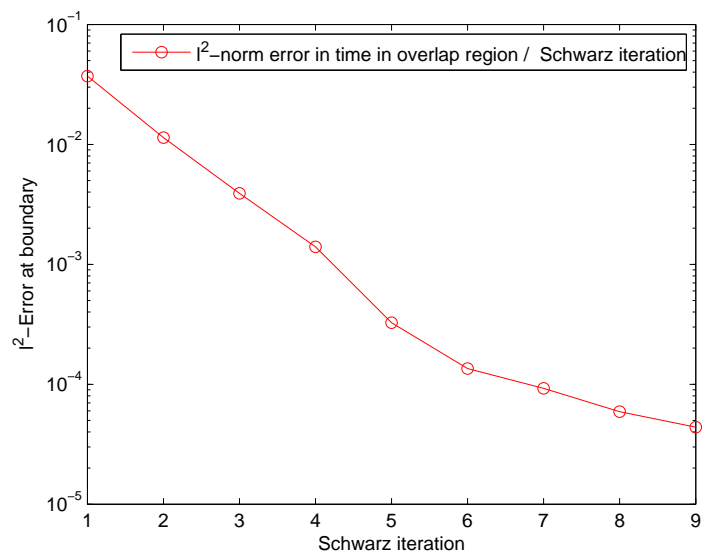

Figure 16: $\ell^{2}$-error in time at the boundary $\left\|v\left(x_{N}, \cdot\right)-w\left(y_{2}, \cdot\right)\right\|_{l^{2}\left(0, T_{f}\right)}$ as a function of Schwarz iterations

which are defined in (10). Recall that $\Lambda_{-1 / 2}^{+}=0$.

$$
E(t)=-E_{0} \exp \left(-\eta\left(t-\frac{T_{f}}{2}\right)^{2}\right) \sin \left(\frac{\pi}{T_{f}} t\right), \quad T_{f}=5, \quad E_{0}=-0.3, \quad \eta=10^{-2}
$$

In addition, the numerical data are given by

- $a=25, c=5$.

- $N=240$ nodes to mesh $(-a, a)=\Omega, 150$ nodes to mesh $\Omega_{1}=(-a, c+\varepsilon)$, and 90 nodes to mesh $(c, a)=\Omega_{2}$. The step size in $\Omega, \Omega_{1}$ and $\Omega_{2}$ is then identical.

- $\left(x_{i}\right)_{i=\{1, \cdots, N\}},\left(y_{i}\right)_{i=\{1, \cdots, M\}}$ denote the mesh nodes respectively in $\Omega_{1}, \Omega_{2}$. The corresponding solutions are denoted $v, w$. The overlap region is reduced to 2 nodes: $x_{N}=y_{2}, x_{N-1}=y_{1}$.

We represent the $\ell^{2}$-norm in time of $v_{N}-w_{2}$, that is $\left(\Delta t \sum_{l=0}^{n}\left|v_{N}^{l}-w_{2}^{l}\right|^{2}\right)^{1 / 2}$ as a function of Schwarz' iteration (see Fig. 17) and at different order $p=0,1,2,3: \partial_{\mathbf{n}}+i \sum_{l=0}^{p} \Lambda_{1 / 2-p / 2}^{+}$. We stop the iteration when 


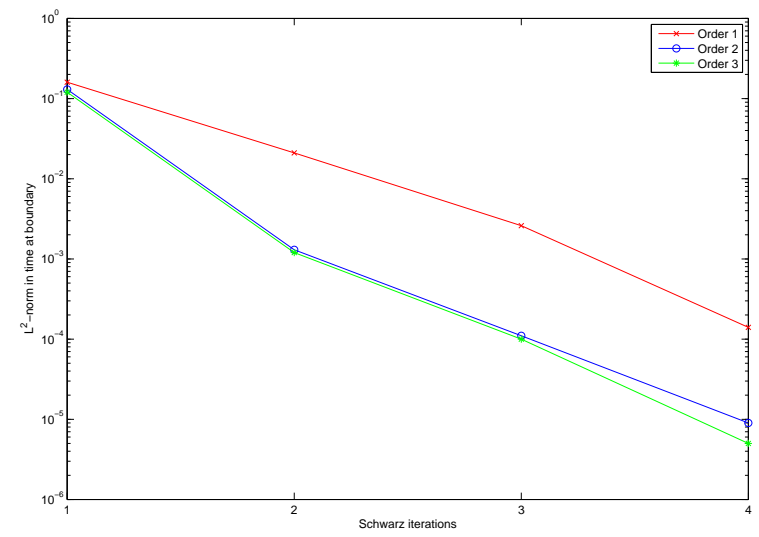

Figure 17: Transmission condition at different orders: $\left(\partial_{\mathbf{n}}+\Lambda_{1 / 2}^{+}\right) u_{1}^{(k)}=\left(\partial_{\mathbf{n}}+i \Lambda_{1 / 2}^{+}\right) u_{2}^{(k-1)},\left(\partial_{\mathbf{n}}+i\left(\Lambda_{1 / 2}^{+}+\Lambda_{0}^{+}\right)\right) u_{1}^{(k)}=$ $\left(\partial_{\mathbf{n}}+i\left(\Lambda_{1 / 2}^{+}+\Lambda_{0}^{+}\right)\right) u_{2}^{(k-1)},\left(\left(\partial_{\mathbf{n}}+i\left(\Lambda_{1 / 2}^{+}+\Lambda_{0}^{+}+\Lambda_{-1}^{+}\right)\right) u_{1}^{(k)}=\left(\partial_{\mathbf{n}}+i\left(\Lambda_{1 / 2}^{+}+\Lambda_{0}^{+}+\Lambda_{-1}^{+}\right)\right) u_{2}^{(k-1)}\right.$

there error reaches $10^{-4}$. We notice that the higher the order of the boundary condition is, the faster is the convergence, although for this simple one-dimensional test the improvement is quite small, as even with $p=0$ we observe a very rapid convergence. Naturally, increasing the order of the conditions would make sense only in higher dimension.

Test 3. This last test consists again of the interaction of an electric field with a two-center molecule using this time 3 subdomains. In that case, in length gauge, the equation writes on $(-a, a)$ :

$$
i \partial_{t} u(x, t)=-\triangle u(x, t)+V_{c}(x) u+x E(t) u(x, t)
$$

with

$$
V_{c}(x)=-\frac{g}{\sqrt{(x-R / 2)^{2}+\varepsilon}}-\frac{g}{\sqrt{(x+R / 2)^{2}+\varepsilon}}
$$

and $R=2$ is the inter-nucleus distance and $\varepsilon$ is a regularizing potential parameter taken equal here to 1 . The initial data is the ground state of the two-center molecule, which is the eigenfunction associated to the smallest eigenvalue to $-\triangle+V_{c}$. We choose $g=0.5$. The electric potential is given by Fig. 18:

$$
E(t)=-E_{0} \exp \left(-\eta\left(t-\frac{T_{f}}{2}\right)^{2}\right) \sin \left(\frac{\pi}{T_{f}} t\right), \quad T_{f}=10, \quad E_{0}=0.15, \quad \eta=10^{-2}
$$

This time we decompose $\Omega$ is 3 subdomains: $\Omega=\Omega_{1} \cup \Omega_{2} \cup \Omega_{3}$ with $\Omega=(-a, a), \Omega_{1}=(-a, c+\varepsilon)$, $\Omega_{2}=(c, d+\varepsilon)$ and $\Omega_{2}=(d, a)$. In addition

- $a=25, c=5, d=15$.

- $N=500$ nodes to mesh $(-a, a)=\Omega, 300$ nodes to mesh $\Omega_{1}=(-a, c+\varepsilon)$, and $M=100$ nodes to mesh $\Omega_{2}=(c, d+\varepsilon)$ as well as $\Omega_{3}=(d, a)$. The step size in $\Omega, \Omega_{1,2,3}$ is then identical.

- $\left(x_{i}\right)_{i=\{1, \cdots, N\}},\left(y_{i}\right)_{i=\{1, \cdots, M\}},\left(z_{i}\right)_{i=\{1, \cdots, M\}}$ denote the nodes respectively in $\Omega_{1}, \Omega_{2}$, $\Omega_{3}$. The corresponding solutions are denoted $v, w$ and $q$. The overlap regions are reduced to 2 nodes: $x_{N}=y_{2}$, $x_{N-1}=y_{1}, y_{M}=z_{2}, y_{M-1}=z_{1}$.

We represent a solution of reference, computed in all $\Omega$, with the reconstructed solution $(v, w, q)$ from $\Omega_{1} \cup \Omega_{2} \cup \Omega_{3}$, at different times $t=T_{f}=10$, as well as the pointwise error in logscale (see Fig. 19). In addition, we represent the convergence graphs for the Schwarz algorithm. More specifically we represent the $\ell^{2}$-norm in time of i) $v_{N}-w_{2}$, that is $\left(\Delta t \sum_{l=0}^{n}\left|v_{N}^{l}-w_{2}^{l}\right|^{2}\right)^{1 / 2}$ and ii) $w_{M}-q_{2}$, that is $\left(\Delta t \sum_{l=0}^{n}\left|w_{M}^{l}-q_{2}^{l}\right|^{2}\right)^{1 / 2}$ (see Fig. 20). 


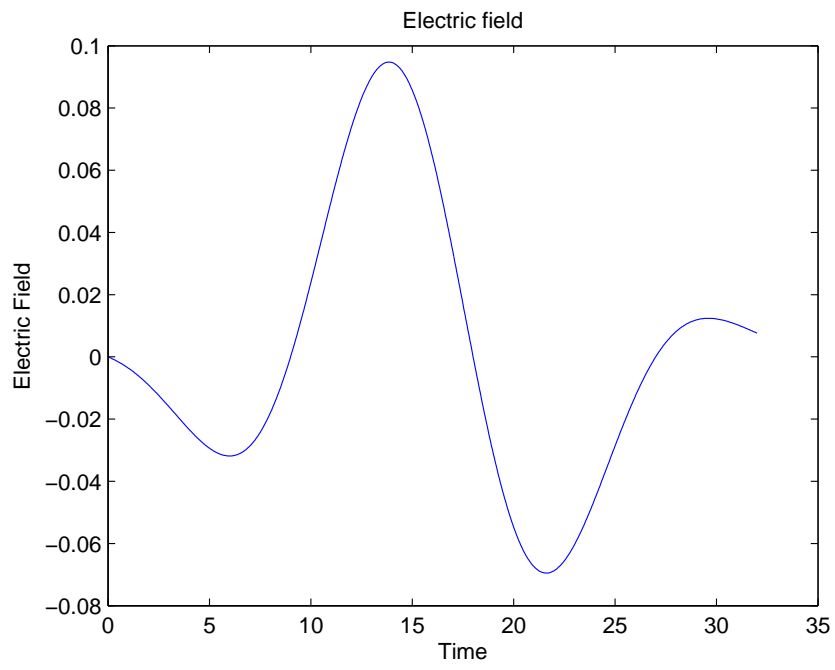

Figure 18: Electric fields as a function of time
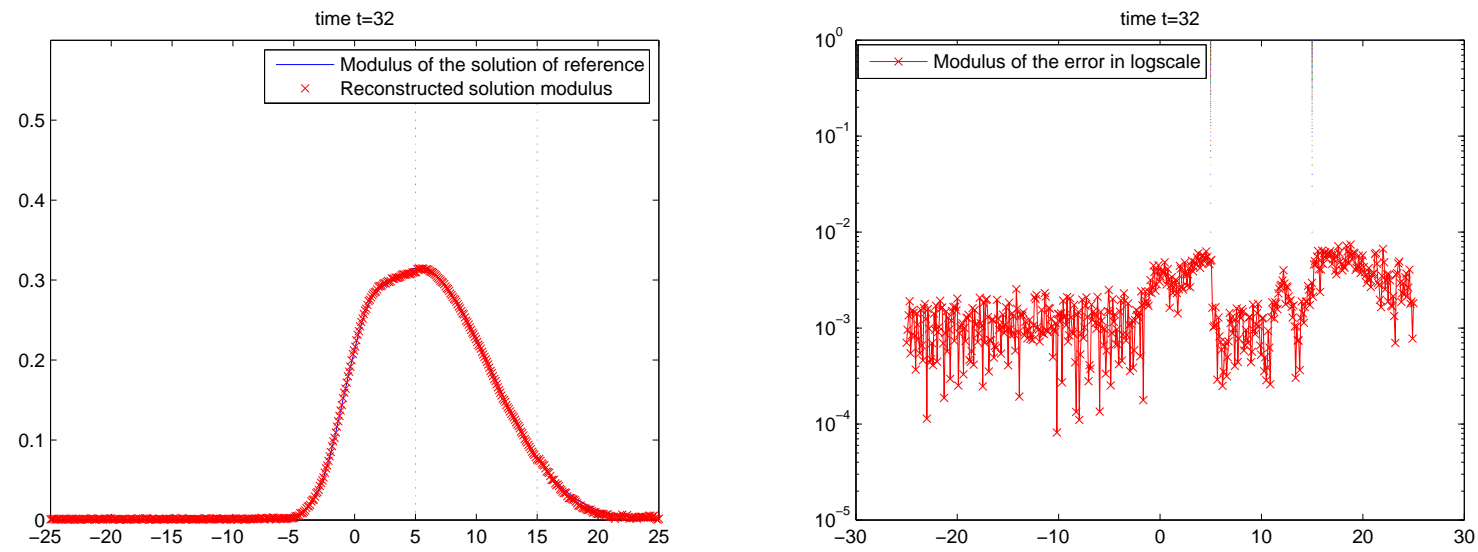

Figure 19: i) Comparison of the modulus of reconstruction solution with the modulus of the solution of reference (computed in all $\Omega$ ) at time $t=T_{f}=10$, ii) pointwise error. Domain interfaces are located at $x=5, x=15$

\section{Conclusion}

This paper was devoted to a general domain decomposition approach allowing to compute much faster than direct methods the solution to TDSE when particles are subject to intense laser fields. The proposed method which is based on an accurate choice of transmission/absorbing boundary conditions and a Schwarz waveform relaxation algorithm, is shown to be accuracy-preserving and efficient compared to the computation in one single domain. In principle, this method makes it possible the simulation of intense laser-molecule interaction involving ionization, recombination and high-order harmonics generation in high dimension. Realistic multidimensional [9] simulations will be proposed in a forthcoming paper.

\section{Acknowledgements.}

The first author thanks the support of the french ANR grants "Bond" (ANR-13-BS01-0009-01) and "BECASIM" (ANR-12-MONU-0007-02). The second and third authors would like to thank NSERC for the 

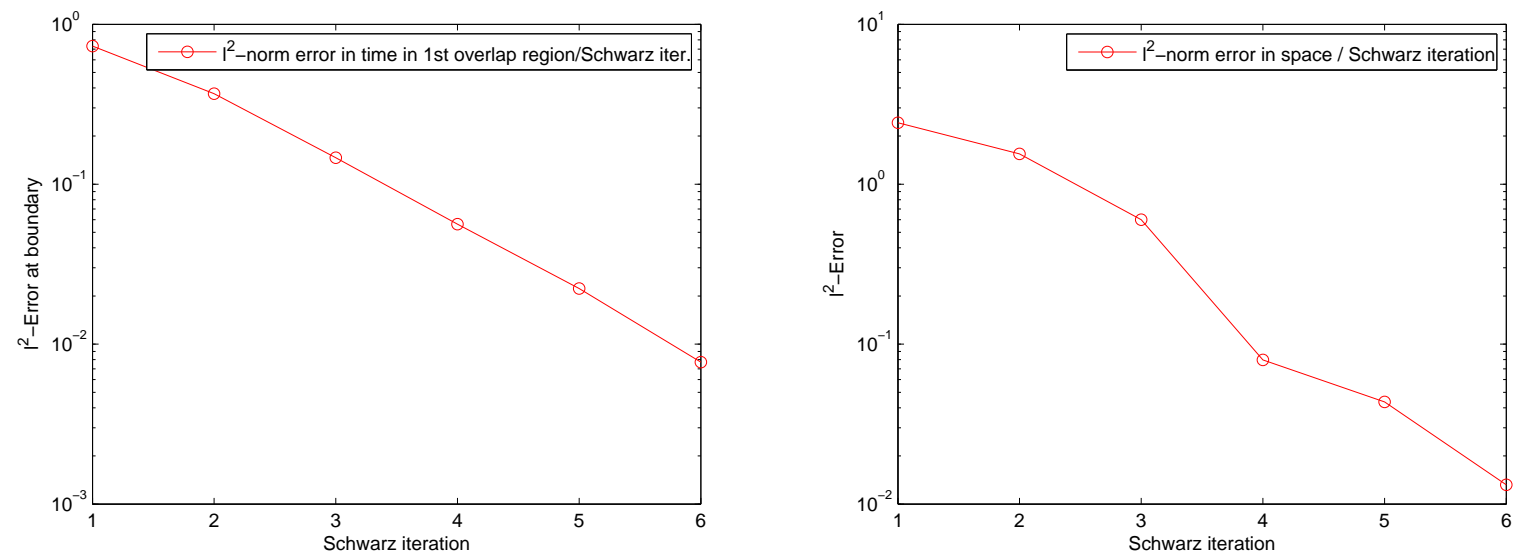

Figure 20: i) $\ell^{2}$-error in time at the boundary (first interface) $\left\|v\left(x_{N}, \cdot\right)-w\left(y_{2}, \cdot\right)\right\|_{l^{2}\left(0, T_{f}\right)}$ as a function of Schwarz iterations, ii) $\ell^{2}$-error in space, as a function of Schwarz iteration at time $t=10$

financial support.

\section{References}

[1] S. Alinhac and P. Gérard. Pseudo-differential operators and the Nash-Moser theorem, volume 82 of Graduate Studies in Mathematics. American Mathematical Society, Providence, RI, 2007. Translated from the 1991 French original by Stephen S. Wilson.

[2] X. Antoine, A. Arnold, C. Besse, M. Ehrhardt, and A. Schädle. A review of transparent and artificial boundary conditions techniques for linear and nonlinear Schrödinger equations. Commun. Comput. Phys., 4(4):729-796, 2008.

[3] X. Antoine, W. Bao, and C. Besse. Computational methods for the dynamics of the nonlinear schrödinger/gross-pitaevskii equations. Comput. Phys. Comm., 184(12):2621-2633, 2013.

[4] X. Antoine, H. Barucq, and A. Bendali. Bayliss-Turkel-like radiation conditions on surfaces of arbitrary shape. J. Math. Anal. Appl., 229(1):184-211, 1999.

[5] X. Antoine and C. Besse. Construction, structure and asymptotic approximations of a microdifferential transparent boundary condition for the linear Schrödinger equation. J. Math. Pures Appl. (9), 80(7):701$738,2001$.

[6] X. Antoine and C. Besse. Unconditionally stable discretization schemes of non-reflecting boundary conditions for the one-dimensional Schrödinger equation. J. Comput. Phys., 188(1):157-175, 2003.

[7] X. Antoine, C. Besse, and P. Klein. Absorbing boundary conditions for the one-dimensional Schrödinger equation with an exterior repulsive potential. J. Comput. Phys., 228(2):312-335, 2009.

[8] X. Antoine, C. Besse, and P. Klein. Absorbing boundary conditions for the two-dimensional Schrödinger equation with an exterior potential. Part I: Construction and a priori estimates. Math. Models Methods Appl. Sci., 22(10):1250026, 38, 2012.

[9] X. Antoine, C. Besse, and P. Klein. Absorbing boundary conditions for the two-dimensional Schrodinger equation with an exterior potential. Part II: Discretization and Numerical Results. Numerische Mathematik, 125(2):191-223, 2013. 
[10] X. Antoine, C. Besse, and J. Szeftel. Towards accurate artificial boundary conditions for nonlinear PDEs through examples. Cubo, 11(4):29-48, 2009.

[11] A. Bandrauk. Molecules in laser fields, chap. 1. M. Dekker, N.Y., 1994.

[12] A. Bandrauk, S. Chelkowski, H. Yu, and E. Constant. Enhanced harmonic generation in extended molecular systems by two-color excitation. Phys. Rev. A, 56:2537-2540, 1997.

[13] A. D. Bandrauk, F. Fillion-Gourdeau, and E. Lorin. Atoms and molecules in intense laser fields: gauge invariance of theory and models. Journal of Physics B-Atomic Molecular and Optical Physics, 46(15), AUG 142013.

[14] V. A. Baskakov and A. V. Popov. Implementation of transparent boundaries for numerical solution of the Schrödinger equation. Wave Motion, 14(2):123-128, 1991.

[15] J.-P. Bérenger. A perfectly matched layer for the absorption of electromagnetic waves. J. Comput. Phys., 114(2):185-200, 1994.

[16] C. Cohen-Tannoudji, J. Dupont-Roc, and G. Grynberg. Atom-photon interactions. Wiley Interscience, N.Y., 1992.

[17] P.-B. Corkum. Plasma perspective on strong-field multiphoton ionization. Phys. Rev. Lett., 71, 1993.

[18] B. Engquist and A. Majda. Absorbing boundary conditions for the numerical simulation of waves. Math. Comp., 31(139):629-651, 1977.

[19] M. Gander and L. Halpern. Optimized schwarz waveform relaxation methods for advection reaction diffusion problems. SIAM J. Num. Anal., 45(2), 2007.

[20] M. J. Gander. Optimized Schwarz methods. SIAM J. Numer. Anal., 44(2):699-731 (electronic), 2006.

[21] M. J. Gander, L. Halpern, and F. Nataf. Optimal Schwarz waveform relaxation for the one dimensional wave equation. SIAM J. Numer. Anal., 41(5):1643-1681, 2003.

[22] T. Hagstrom, R. P. Tewarson, and A. Jazcilevich. Numerical experiments on a domain decomposition algorithm for nonlinear elliptic boundary value problems. Appl. Math. Lett., 1(3):299-302, 1988.

[23] L. Halpern and J. Rauch. Error analysis for absorbing boundary conditions. Numer. Math., 51(4):459$467,1987$.

[24] L. Halpern and J. Szeftel. Optimized and quasi-optimal Schwarz waveform relaxation for the onedimensional Schrödinger equation. Math. Models Methods Appl. Sci., 20(12):2167-2199, 2010.

[25] L. Hörmander. The analysis of linear partial differential operators. III. Classics in Mathematics. Springer, Berlin, 2007. Pseudo-differential operators.

[26] S. Jiang and L. Greengard. Fast evaluation of nonreflecting boundary conditions for the Schrödinger equation in one dimension. Comput. Math. Appl., 47(6-7):955-966, 2004.

[27] G. L. Kamta and A. D. Bandrauk. High-order harmonic generation from two-center molecules: Timeprofile analysis of nuclear contributions. Phys. Rev. A, 70(1):011404-1-011404-4, 2004.

[28] M. Lewenstein, Ph. Balcou, M.Yu. Ivanov, A. L'Huillier, and P.B. Corkum. Theory of high-harmonic generation by low-frequency laser fields. Phys. Rev. A, 49(3):2117-2132, 1994.

[29] E. Lorin, S. Chelkowski, and A. Bandrauk. A numerical Maxwell-Schrödinger model for laser-matter interaction and propagation. Comput. Phys. Comm., 177(12):908-932, 2007.

[30] E. Lorin, S. Chelkowski, and A. Bandrauk. Attosecond pulse generation from aligned molecules dynamics and propagation in $\mathrm{H}_{2}^{+}$. New J. Phys., 10(025033):21pp, 2008. 
[31] E. Lorin, S. Chelkowski, and A. D. Bandrauk. Mathematical modeling of boundary conditions for lasermolecule time-dependent Schrödinger equations and some aspects of their numerical computation - onedimensional case. Numer. Methods Partial Differential Equations, 25(1):110-136, 2009.

[32] D. B. Milosevic, G. G. Paulus, D. Bauer, and W. Becker. Above-threshold ionization by few-cycle pulses. J. of Phys. B: Atomic, Molecular and Optical Physics, 39(14):R203-R262, 2006.

[33] J. C. Strikwerda. Finite difference schemes and partial differential equations. Society for Industrial and Applied Mathematics (SIAM), Philadelphia, PA, second edition, 2004.

[34] J. Szeftel. Absorbing boundary conditions for one-dimensional nonlinear Schrödinger equations. Numer. Math., 104(1):103-127, 2006. 